\title{
Certain new weighted estimates proposing generalized proportional fractional operator in another sense
}

\author{
Thabet Abdeljawad ${ }^{1,2,3}$, Saima Rashid ${ }^{4}$, A.A. El-Deeb ${ }^{5}$, Zakia Hammouch ${ }^{6}$ and Yu-Ming Chu C $^{7,8^{*}}$
}

\section{"Correspondence:}

chuyuming2005@126.com

${ }^{7}$ Department of Mathematics,

Huzhou University, Huzhou, China

${ }^{8}$ Hunan Provincial Key Laboratory of

Mathematical Modeling and

Analysis in Engineering, Changsha

University of Science \& Technology,

Changsha, China

Full list of author information is

available at the end of the article

\section{Springer}

\begin{abstract}
The present work investigates the applicability and effectiveness of generalized proportional fractional integral $(\mathcal{G P F})$ ) operator in another sense. We aim to derive novel weighted generalizations involving a family of positive functions $n(n \in \mathbb{N})$ for this recently proposed operator. As applications of this operator, we can generate notable outcomes for Riemann-Liouville $(\mathcal{R} \mathcal{L})$ fractional, generalized $\mathcal{R} \mathcal{L}$-fractional operator, conformable fractional operator, Katugampola fractional integral operator, and Hadamard fractional integral operator by changing the domain. The proposed strategy is vivid, explicit, and it can be used to derive new solutions for various fractional differential equations applied in mathematical physics. Certain remarkable consequences of the main theorems are also figured.
\end{abstract}

MSC: 26D15; 26A33; 26A51

Keywords: Integral inequality; Generalized proportional fractional integral with respect to another function; Increasing and decreasing functions

\section{Introduction}

Fractional calculus [1-20] is genuinely viewed to be the real-world framework, and it has wide applications in mathematics, physics, biology, medicine, and many other natural and social sciences [21-35], for instance, a correspondence structure that contains indulgent interfacing, dependent parts that are used to accomplish a bound together with a goal of transmitting and getting signals, can be depicted using complex framework models [3643]. This structure is considered as a stunning framework, and the units that make the entire system are seen as the centers of the complex framework. An attracting particularization of this field is that there are various fractional operators, and this allows the scientists to pick out the most suitable operator for the purpose of displaying the issue under scrutiny [44-47]. Moreover, due to its effortlessness in applications, analysts have paid more noteworthy enthusiasm to fractional operators without singular kernels [4850], after which numerous articles considering these sorts of fractional operators have as of late become visible. These methods had been created by various mathematicians with a

(c) The Author(s) 2020. This article is licensed under a Creative Commons Attribution 4.0 International License, which permits use sharing, adaptation, distribution and reproduction in any medium or format, as long as you give appropriate credit to the original author(s) and the source, provide a link to the Creative Commons licence, and indicate if changes were made. The images or other third party material in this article are included in the article's Creative Commons licence, unless indicated otherwise in a credit line to the material. If material is not included in the article's Creative Commons licence and your intended use is not permitted by statutory regulation or exceeds the permitted use, you will need to obtain permission directly from the copyright holder. To view a copy of this licence, visit http://creativecommons.org/licenses/by/4.0/. 
scarcely explicit definition, as in the case of $\mathcal{R} \mathcal{L}$, Weyl, Erdelyi-Kober, Hadamard integral, Liouville and Katugampola fractional operator [44-46, 48-52].

Recently, Jarad et al. [53] explored the idea of $\mathcal{G P F} \mathcal{I}$ operators which have been applied to characterize certain probability density functions and have fertile applications in statistics. In [54, 55], Rashid et al. proposed a different novel fractional approach having an exponential function in its kernel which comes into existence in the theory of fractional calculus, which is known as $\mathcal{G P F} \mathcal{I}$ operators in another sense. The significant characterization of the $\mathcal{G P \mathcal { F }}$ operator in another sense is that it can discover the bulk of complex problems in one direction, and then again the generalized proportional fractional derivative in the sense of another function can catch various sorts of complexities, hence assembling these two ideas can help us to comprehend the complexities of existing nature in a vastly improved manner. The $\mathcal{G P F \mathcal { I }}$ operators have captivated the interest of many researchers from several areas of science. This novel concept provides an avenue for interested readers towards various scientific fields of research, including control theory, engineering, fluid dynamics, meteorology, analysis, aerodynamics, and many more.

Inequalities are an important part of the whole field of mathematical research [56-69], fractional calculus can be applied on many equalities and inequalities that have been explored by many authors, such as the Hardy, Ostrowski, Gagliardo-Nirenberg, Olsen, and trapezoidal-type inequalities, which are utilized in imperative significant systems among scientists and amass prolific utilitarian applications in different regions of science [7072]. Fractional integral inequalities have potential applications in several areas of science, such as technology, mathematics, chemistry, plasma physics, and so on. Particularly, we bring up the initial value problem, the stability of linear transformation, integrodifferential equations, and transform equations [44-46]. Such utilizations of fractional integral operators constrained us to show the speculation by utilizing a group of $n$ positive functions including $\mathcal{G P} \mathcal{F} \mathcal{I}$ operators in another sense.

In the present study, we introduce new weighted versions of several generalizations and enunciate a new generalized fractional proportional integrals, which we name $\mathcal{G P \mathcal { F }}$ operator in another sense. To be more precise, we establish a new version for a class of family of $n(n \in \mathbb{N})$ continuous positive decreasing functions in the frame of the $\mathcal{G P} \mathcal{F} \mathcal{I}$ operator in another sense and also provide some of its trendy splendor consequences observing Remark 2.2. New findings are introduced and new theorems which relate to $\mathcal{G P \mathcal { F }}$ and $\mathcal{R} \mathcal{L}$ are derived that correlate with the earlier results.

\section{Prelude}

This section consists of some useful preliminaries from fractional calculus used in our subsequent discussion. The major subtleties are given in the monograph by Kilbas et al. [73].

Now, we demonstrate a novel fractional operator which is known as the $\mathcal{G P F} \mathcal{I}$ operator of a function in another sense proposed by Jarad et al. [54] and Rashid et al. [55], independently.

Definition 2.1 (see [54,55]) Let $\zeta>0,\left(\mu_{1}, \mu_{2}\right)\left(-\infty \leq \mu_{1}<\mu_{2} \leq \infty\right)$ be a finite or infinite real interval, and $\varphi\left(y_{1}\right)$ an increasing and positive monotone function on $\left(\mu_{1}, \mu_{2}\right]$. Then the left- and right-sided $\mathcal{G P \mathcal { F }}$ operators of a function $\mathcal{P}$ with respect to another function 
$\varphi$ of order $\zeta>0$ are defined by (2.1) and (2.2) as follows:

$$
\begin{array}{ll}
{ }^{\varphi} \mathcal{K}_{\mu_{1}}^{\zeta, \sigma} \mathcal{P}(\varsigma)=\frac{1}{\sigma^{\zeta} \Gamma(\zeta)} \int_{\mu_{1}}^{\varsigma} \frac{\exp \left[\frac{\sigma-1}{\sigma}\left(\varphi(\varsigma)-\varphi\left(y_{1}\right)\right)\right]}{\left(\varphi(\varsigma)-\varphi\left(y_{1}\right)\right)^{1-\zeta}} \varphi^{\prime}\left(y_{1}\right) \mathcal{P}\left(y_{1}\right) d y_{1} & \left(\mu_{1}<\varsigma\right), \\
{ }^{\varphi} \mathcal{K}_{\mu_{2}}^{\zeta, \sigma} \mathcal{P}(\varsigma)=\frac{1}{\sigma^{\zeta} \Gamma(\zeta)} \int_{\varsigma}^{\mu_{2}} \frac{\exp \left[\frac{\sigma-1}{\sigma}\left(\varphi\left(y_{1}\right)-\varphi(\varsigma)\right)\right]}{\left(\varphi\left(y_{1}\right)-\varphi(\varsigma)\right)^{1-\zeta}} \varphi^{\prime}\left(y_{1}\right) \mathcal{P}\left(y_{1}\right) d y_{1} & \left(\varsigma<\mu_{2}\right),
\end{array}
$$

where the proportionality index $\sigma \in(0,1], \varsigma \in \mathcal{C}$ with $\mathfrak{R}(\varsigma)>0$ and $\Gamma(\varsigma)=\int_{0}^{\infty} y_{1}^{\varsigma-1} e^{-y_{1}} d y_{1}$ is the Gamma function.

Remark 2.2 From Definition 2.1 we clearly see that

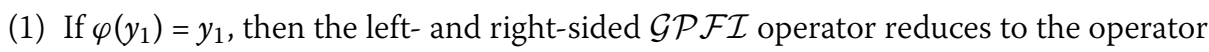
given in [53].

(2) If $\sigma=1$, then the left- and right-sided generalized $\mathcal{R} \mathcal{L}$-fractional integral operator reduces to the operator defined in [73].

(3) If $\sigma=1$ and $\varphi\left(y_{1}\right)=y_{1}$, then the left- and right-sided $\mathcal{R} \mathcal{L}$-fractional integral operator reduces to the operator given in [73].

(4) If $\varphi\left(y_{1}\right)=\ln y_{1}$, then the left- and right-sided generalized proportional Hadamard fractional integral operator reduces to the operator given in [74].

(5) If $\varphi\left(y_{1}\right)=\ln y_{1}$ and $\sigma=1$, then the left- and right-sided Hadamard fractional integral operator reduces to the operator defined in [75].

Next, we present the definition of the one-sided $\mathcal{G P F} \mathcal{I}$ operator with respect to another function $\varphi$.

Definition 2.3 Let $\left(\mu_{1}, \mu_{2}\right)\left(-\infty \leq \mu_{1}<\mu_{2} \leq \infty\right)$ be a finite or infinite real interval, $\zeta>0$, and $\varphi\left(y_{1}\right)$ an increasing and positive monotone function on $\left(\mu_{1}, \mu_{2}\right]$. Then the one-sided $\mathcal{G P \mathcal { F }}$ operator of a function $\mathcal{P}$ with respect to another function $\varphi$ of order $\zeta>0$ is defined by

$$
{ }^{\varphi} \mathcal{K}_{0^{+}}^{\zeta, \sigma} \mathcal{P}(\varsigma)=\frac{1}{\sigma^{\zeta} \Gamma(\zeta)} \int_{0}^{\varsigma} \frac{\exp \left[\frac{\sigma-1}{\sigma}\left(\varphi(\varsigma)-\varphi\left(y_{1}\right)\right)\right]}{\left(\varphi(\varsigma)-\varphi\left(y_{1}\right)\right)^{1-\zeta}} \varphi^{\prime}\left(y_{1}\right) \mathcal{P}\left(y_{1}\right) d y_{1} \quad(\varsigma>0)
$$

\section{Main results}

In what follows, we suppose that $\varphi\left(y_{1}\right)$ a continuous, increasing, and positive function on $[0, \infty)$ with $\varphi(0)=0$.

Throughout this paper, we suppose that $\varphi\left(y_{1}\right)$ is an increasing, positive monotone function on $[0, \infty)$, and also $\varphi\left(y_{1}\right)$ is continuous on $[0, \infty)$ with $\varphi(0)=0$.

Next, we provide certain inequalities for a class of family of continuous, positive and decreasing functions via $\mathcal{G P} \mathcal{F} \mathcal{I}$ defined in (2.3).

Theorem 3.1 Assume that there are two positive and continuous functions $\mathcal{P}$ and $\mathcal{Q}$ defined on $[0, \infty)$ with the assumption that $\mathcal{R}:[0, \infty) \rightarrow \mathbb{R}$ is positive and continuous. Then for all $\varsigma>0$, the following inequality holds:

$$
\begin{gathered}
{ }^{\varphi} \mathcal{K}_{0^{+}}^{\zeta, \sigma}\left[\mathcal{R}(\varsigma) \mathcal{P}^{\beta+\rho}(\varsigma)\right]^{\varphi} \mathcal{K}_{0^{+}}^{\zeta, \sigma}\left[\mathcal{R}(\varsigma) \mathcal{Q}^{\rho}(\varsigma) \mathcal{P}^{\eta}(\varsigma)\right] \\
\geq{ }^{\varphi} \mathcal{K}_{0^{+}}^{\zeta, \sigma}\left[\mathcal{R}(\varsigma) \mathcal{P}^{\rho+\eta}(\varsigma)\right]^{\varphi} \mathcal{K}_{0^{+}}^{\zeta, \sigma}\left[\mathcal{R}(\varsigma) \mathcal{Q}^{\rho}(\varsigma) \mathcal{P}^{\beta}(\varsigma)\right],
\end{gathered}
$$

where $\beta \geq \eta>0, \rho>0, \sigma \in(0,1]$, and $\zeta \in \mathcal{C}(\Re(\zeta)>0)$. 
Proof Since $\mathcal{P}$ and $\mathcal{Q}$ are positive and continuous functions defined on $[0, \infty)$, therefore for all $y_{1}, z_{1} \in(0, \varsigma), \varsigma>0$ and for any $\rho>0, \beta \geq \eta>0$, we have

$$
\left(\mathcal{Q}^{\rho}\left(z_{1}\right) \mathcal{P}^{\rho}\left(y_{1}\right)-\mathcal{Q}^{\rho}\left(y_{1}\right) \mathcal{P}^{\rho}\left(z_{1}\right)\right)\left(\mathcal{P}^{\beta-\eta}\left(y_{1}\right)-\mathcal{P}^{\beta-\eta}\left(z_{1}\right)\right) \geq 0 .
$$

It follows that

$$
\begin{aligned}
& \mathcal{Q}^{\rho}\left(z_{1}\right) \mathcal{P}^{\rho+\beta-\eta}\left(y_{1}\right)+\mathcal{Q}^{\rho}\left(y_{1}\right) \mathcal{P}^{\rho+\beta-\eta}\left(z_{1}\right) \\
& \geq \mathcal{Q}^{\rho}\left(z_{1}\right) \mathcal{P}^{\rho}\left(y_{1}\right) \mathcal{P}^{\beta-\eta}\left(z_{1}\right)+\mathcal{Q}^{\rho}\left(y_{1}\right) \mathcal{P}^{\rho}\left(z_{1}\right) \mathcal{P}^{\beta-\eta}\left(y_{1}\right) .
\end{aligned}
$$

Multiplying both sides of (3.2) by $\frac{1}{\sigma^{\zeta} \Gamma(\zeta)} \frac{\exp \left[\frac{\sigma-1}{\sigma}\left(\varphi(\varsigma)-\varphi\left(y_{1}\right)\right)\right]}{\left(\varphi(\varsigma)-\varphi\left(y_{1}\right)\right)^{1-\zeta}} \varphi^{\prime}\left(y_{1}\right) \mathcal{R}\left(y_{1}\right) \mathcal{P}^{\eta}\left(y_{1}\right)$ and then integrating the obtained inequality with respect to $y_{1}$ over $(0, \varsigma)$, we get

$$
\begin{aligned}
& \frac{\mathcal{Q}^{\rho}\left(z_{1}\right)}{\sigma^{\zeta} \Gamma(\zeta)} \int_{0}^{\varsigma} \frac{\exp \left[\frac{\sigma-1}{\sigma}\left(\varphi(\varsigma)-\varphi\left(y_{1}\right)\right)\right]}{\left(\varphi(\varsigma)-\varphi\left(y_{1}\right)\right)^{1-\zeta}} \varphi^{\prime}\left(y_{1}\right) \mathcal{R}\left(y_{1}\right) \mathcal{P}^{\eta}\left(y_{1}\right) \mathcal{P}^{\rho+\beta-\eta}\left(y_{1}\right) d y_{1} \\
& \quad+\frac{\mathcal{P}^{\rho+\beta-\eta}\left(z_{1}\right)}{\sigma^{\zeta} \Gamma(\zeta)} \int_{0}^{\varsigma} \frac{\exp \left[\frac{\sigma-1}{\sigma}\left(\varphi(\varsigma)-\varphi\left(y_{1}\right)\right)\right]}{\left(\varphi(\varsigma)-\varphi\left(y_{1}\right)\right)^{1-\zeta}} \varphi^{\prime}\left(y_{1}\right) \mathcal{R}\left(y_{1}\right) \mathcal{P}^{\eta}\left(y_{1}\right) \mathcal{Q}^{\rho}\left(y_{1}\right) d y_{1} \\
& \geq \frac{\mathcal{Q}^{\rho}\left(z_{1}\right) \mathcal{P}^{\beta-\eta}\left(z_{1}\right)}{\sigma^{\zeta} \Gamma(\zeta)} \int_{0}^{\varsigma} \frac{\exp \left[\frac{\sigma-1}{\sigma}\left(\varphi(\varsigma)-\varphi\left(y_{1}\right)\right)\right]}{\left(\varphi(\varsigma)-\varphi\left(y_{1}\right)\right)^{1-\zeta}} \varphi^{\prime}\left(y_{1}\right) \mathcal{R}\left(y_{1}\right) \mathcal{P}^{\eta}\left(y_{1}\right) \mathcal{P}^{\rho}\left(y_{1}\right) d y_{1} \\
& \quad+\frac{\mathcal{P}^{\rho}\left(z_{1}\right)}{\sigma^{\zeta} \Gamma(\zeta)} \int_{0}^{\varsigma} \frac{\exp \left[\frac{\sigma-1}{\sigma}\left(\varphi(\varsigma)-\varphi\left(y_{1}\right)\right)\right]}{\left(\varphi(\varsigma)-\varphi\left(y_{1}\right)\right)^{1-\zeta}} \varphi^{\prime}\left(y_{1}\right) \mathcal{R}\left(y_{1}\right) \mathcal{P}^{\eta}\left(y_{1}\right) \mathcal{Q}^{\rho}\left(y_{1}\right) \mathcal{P}^{\beta-\eta}\left(y_{1}\right) d y_{1} .
\end{aligned}
$$

In view of Definition 2.3, we have

$$
\begin{aligned}
& \mathcal{Q}^{\rho}\left(z_{1}\right)^{\varphi} \mathcal{K}_{0^{+}}^{\zeta, \sigma}\left[\mathcal{R}(\varsigma) \mathcal{P}^{\beta+\rho}(\varsigma)\right]+\mathcal{P}^{\rho+\beta-\eta}\left(z_{1}\right)^{\varphi} \mathcal{K}_{0^{+}}^{\zeta, \sigma}\left[\mathcal{R}(\varsigma) \mathcal{Q}^{\rho}(\varsigma) \mathcal{P}^{\eta}(\varsigma)\right] \\
& \geq \mathcal{Q}^{\rho}\left(z_{1}\right) \mathcal{P}^{\beta-\eta}\left(z_{1}\right)^{\varphi} \mathcal{K}_{0^{+}}^{\zeta, \sigma}\left[\mathcal{R}(\varsigma) \mathcal{P}^{\rho+\eta}\left(y_{1}\right)\right]+\mathcal{P}^{\rho}\left(z_{1}\right) \mathcal{K}_{0^{+}}^{\zeta, \sigma}\left[\mathcal{R}(\varsigma) \mathcal{Q}^{\rho}(\varsigma) \mathcal{P}^{\beta}(\varsigma)\right] .
\end{aligned}
$$

Again, if we multiply both sides of (3.3) by $\frac{1}{\sigma^{\zeta} \Gamma(\zeta)} \frac{\exp \left[\frac{\sigma-1}{\sigma}\left(\varphi(\varsigma)-\varphi\left(z_{1}\right)\right)\right]}{\left(\varphi(\varsigma)-\varphi\left(z_{1}\right)\right)^{1-\zeta}} \varphi^{\prime}\left(z_{1}\right) \mathcal{R}\left(z_{1}\right) \mathcal{P}^{\eta}\left(z_{1}\right)$ and integrate the obtained inequality with respect to $z_{1}$ over $(0, \varsigma)$, then, by using $(2.3)$, we obtain

$$
\begin{aligned}
&{ }^{\varphi} \mathcal{K}_{0^{+}}^{\zeta, \sigma} {\left[\mathcal{R}(\varsigma) \mathcal{Q}^{\rho}(\varsigma) \mathcal{P}^{\eta}(\varsigma)\right]^{\varphi} \mathcal{K}_{0^{+}}^{\zeta, \sigma}\left[\mathcal{R}(\varsigma) \mathcal{P}^{\rho+\beta}(\varsigma)\right] } \\
&+{ }^{\varphi} \mathcal{K}_{0^{+}}^{\zeta, \sigma}\left[\mathcal{R}(\varsigma) \mathcal{P}^{\rho+\beta}(\varsigma)\right]^{\varphi} \mathcal{K}_{0^{+}}^{\zeta, \sigma}\left[\mathcal{R}(\varsigma) \mathcal{Q}^{\rho}(\varsigma) \mathcal{P}^{\eta}(\varsigma)\right] \\
& \geq{ }^{\varphi} \mathcal{K}_{0^{+}}^{\zeta, \sigma}\left[\mathcal{R}(\varsigma) \mathcal{Q}^{\rho}(\varsigma) \mathcal{P}^{\beta}(\varsigma)\right]^{\varphi} \mathcal{K}_{0^{+}}^{\zeta, \sigma}\left[\mathcal{R}(\varsigma) \mathcal{P}^{\rho+\eta}(\varsigma)\right] \\
&{ }^{\varphi}{ }^{\varphi} \mathcal{K}_{0^{+}}^{\zeta, \sigma}\left[\mathcal{R}(\varsigma) \mathcal{P}^{\rho+\eta}(\varsigma)\right]^{\varphi} \mathcal{K}_{0^{+}}^{\zeta, \sigma}\left[\mathcal{R}(\varsigma) \mathcal{Q}^{\rho}(\varsigma) \mathcal{P}^{\beta}(\varsigma)\right],
\end{aligned}
$$

which gives the desired inequality (3.1).

Theorem 3.2 Assume that there are two positive and continuous functions $\mathcal{P}$ and $\mathcal{Q}$ defined on $[0, \infty)$ with the assumption that $\mathcal{R}:[0, \infty) \rightarrow \mathbb{R}$ is positive and continuous. Then for all $\varsigma>0$, we have the following inequality:

$$
\begin{aligned}
{ }^{\varphi} \mathcal{K}_{0^{+}}^{\zeta, \sigma} & {\left[\mathcal{R}(\varsigma) \mathcal{P}^{\rho+\beta}(\varsigma)\right]^{\varphi} \mathcal{K}_{0^{+}}^{\vartheta, \sigma}\left[\mathcal{R}(\varsigma) \mathcal{Q}^{\rho}(\varsigma) \mathcal{P}^{\eta}(\varsigma)\right] } \\
& +{ }^{\varphi} \mathcal{K}_{0^{+}}^{\zeta, \sigma}\left[\mathcal{R}(\varsigma) \mathcal{Q}^{\rho}(\varsigma) \mathcal{P}^{\eta}(\varsigma)\right]^{\varphi} \mathcal{K}_{0^{+}}^{\vartheta, \sigma}\left[\mathcal{R}(\varsigma) \mathcal{P}^{\rho+\beta}(\varsigma)\right]
\end{aligned}
$$




$$
\begin{aligned}
\geq & { }^{\varphi} \mathcal{K}_{0^{+}}^{\zeta, \sigma}\left[\mathcal{R}(\varsigma) \mathcal{P}^{\rho+\eta}(\varsigma)\right]^{\varphi} \mathcal{K}_{0^{+}}^{\vartheta, \sigma}\left[\mathcal{R}(\varsigma) \mathcal{Q}^{\rho}(\varsigma) \mathcal{P}^{\beta}(\varsigma)\right] \\
& +{ }^{\varphi} \mathcal{K}_{0^{+}}^{\zeta, \sigma}\left[\mathcal{R}(\varsigma) \mathcal{Q}^{\rho}(\varsigma) \mathcal{P}^{\beta}(\zeta)\right]^{\varphi} \mathcal{K}_{0^{+}}^{\zeta, \sigma}\left[\mathcal{R}(\varsigma) \mathcal{P}^{\rho+\eta}(\varsigma)\right]
\end{aligned}
$$

where $\beta \geq \eta>0, \rho>0, \sigma \in(0,1], \zeta \in \mathcal{C}, \mathfrak{R}(\zeta)>0$.

Proof Multiplying both sides of (3.3) by $\frac{1}{\sigma^{\vartheta} \Gamma(\vartheta)} \frac{\exp \left[\frac{\sigma-1}{\sigma}\left(\varphi(\varsigma)-\varphi\left(z_{1}\right)\right)\right]}{\left(\varphi(\varsigma)-\varphi\left(z_{1}\right)\right)^{1-\vartheta}} \varphi^{\prime}\left(z_{1}\right) \mathcal{R}\left(z_{1}\right) \mathcal{P}^{\eta}\left(z_{1}\right)$, where $\vartheta, \eta>0$ and $z_{1} \in(0, \varsigma), \varsigma>0$, and then integrating the obtained inequality with respect to $z_{1}$ over $(0, \varsigma)$, we have

$$
\begin{aligned}
& \frac{\varphi \mathcal{K}_{0^{+}}^{\zeta, \sigma}\left[\mathcal{R}(\varsigma) \mathcal{P}^{\rho+\beta}(\varsigma)\right]}{\sigma^{\vartheta} \Gamma(\vartheta)} \int_{0}^{\varsigma} \frac{\exp \left[\frac{\sigma-1}{\sigma}\left(\varphi(\varsigma)-\varphi\left(z_{1}\right)\right)\right]}{\left(\varphi(\varsigma)-\varphi\left(z_{1}\right)\right)^{1-\vartheta}} \varphi^{\prime}\left(z_{1}\right) \mathcal{R}\left(z_{1}\right) \mathcal{P}^{\eta}\left(z_{1}\right) \mathcal{Q}^{\rho}\left(z_{1}\right) d z_{1} \\
& \quad+\frac{\varphi \mathcal{K}_{0^{+}}^{\zeta, \sigma}\left[\mathcal{R}(\varsigma) \mathcal{Q}^{\rho}(\varsigma) \mathcal{P}^{\eta}(\varsigma)\right]}{\sigma^{\vartheta} \Gamma(\vartheta)} \\
& \quad \times \int_{0}^{\varsigma} \frac{\exp \left[\frac{\sigma-1}{\sigma}\left(\varphi(\varsigma)-\varphi\left(z_{1}\right)\right)\right]}{\left(\varphi(\varsigma)-\varphi\left(z_{1}\right)\right)^{1-\vartheta}} \varphi^{\prime}\left(z_{1}\right) \mathcal{R}\left(z_{1}\right) \mathcal{P}^{\eta}\left(z_{1}\right) \mathcal{P}^{\rho+\beta-\eta}\left(z_{1}\right) d z_{1} \\
& \geq \frac{{ }_{\mathcal{K}} \mathcal{K}_{0^{+}}^{\zeta, \sigma}\left[\mathcal{R}(\varsigma) \mathcal{P}^{\rho+\eta}(\varsigma)\right]}{\sigma^{\vartheta} \Gamma(\vartheta)} \\
& \quad \times \int_{0}^{\varsigma} \frac{\exp \left[\frac{\sigma-1}{\sigma}\left(\varphi(\varsigma)-\varphi\left(z_{1}\right)\right)\right]}{\left(\varphi(\varsigma)-\varphi\left(z_{1}\right)\right)^{1-\vartheta}} \varphi^{\prime}\left(z_{1}\right) \mathcal{R}\left(z_{1}\right) \mathcal{P}^{\eta}\left(z_{1}\right) \mathcal{Q}^{\rho}\left(z_{1}\right) \mathcal{P}^{\beta-\eta}\left(z_{1}\right) d z_{1} \\
& \quad+\frac{\varphi \mathcal{K}_{0^{+}}^{\zeta, \sigma}\left[\mathcal{R}(\varsigma) \mathcal{Q}^{\rho}(\varsigma) \mathcal{P}^{\beta}(\varsigma)\right]}{\sigma^{\vartheta} \Gamma(\vartheta)} \\
& \quad \times \int_{0}^{\varsigma} \frac{\exp \left[\frac{\sigma-1}{\sigma}\left(\varphi(\varsigma)-\varphi\left(z_{1}\right)\right)\right]}{\left(\varphi(\varsigma)-\varphi\left(z_{1}\right)\right)^{1-\vartheta}} \varphi^{\prime}\left(z_{1}\right) \mathcal{R}\left(z_{1}\right) \mathcal{P}^{\eta}\left(z_{1}\right) \mathcal{P}^{\rho}\left(z_{1}\right) d z_{1} .
\end{aligned}
$$

It follows from Definition 2.3 that

$$
\begin{aligned}
&{ }^{\varphi} \mathcal{K}_{0^{+}}^{\zeta, \sigma} {\left[\mathcal{R}(\varsigma) \mathcal{P}^{\rho+\beta}(\varsigma)\right]^{\varphi} \mathcal{K}_{0^{+}}^{\vartheta, \sigma}\left[\mathcal{R}(\varsigma) \mathcal{Q}^{\rho}(\varsigma) \mathcal{P}^{\eta}(\varsigma)\right] } \\
&+{ }^{\varphi} \mathcal{K}_{0^{+}}^{\zeta, \sigma}\left[\mathcal{R}(\varsigma) \mathcal{Q}^{\rho}(\varsigma) \mathcal{P}^{\eta}(\varsigma)\right]^{\varphi} \mathcal{K}_{0^{+}}^{\vartheta, \sigma}\left[\mathcal{R}(\varsigma) \mathcal{P}^{\rho+\beta}(\varsigma)\right] \\
& \geq{ }^{\varphi} \mathcal{K}_{0^{+}}^{\zeta, \sigma}\left[\mathcal{R}(\varsigma) \mathcal{P}^{\rho+\eta}(\varsigma)\right]^{\varphi} \mathcal{K}_{0^{+}}^{\vartheta, \sigma}\left[\mathcal{R}(\varsigma) \mathcal{Q}^{\rho}(\varsigma) \mathcal{P}^{\beta}(\varsigma)\right] \\
&{ }^{\varphi}{ }^{\mathcal{K}_{0^{+}}^{\zeta, \sigma}}\left[\mathcal{R}(\varsigma) \mathcal{Q}^{\rho}(\varsigma) \mathcal{P}^{\beta}(\varsigma)\right]^{\varphi} \mathcal{K}_{0^{+}}^{\vartheta, \sigma}\left[\mathcal{R}(\varsigma) \mathcal{P}^{\rho+\eta}(\varsigma)\right],
\end{aligned}
$$

which is the desired inequality (3.4).

Remark 3.3 Applying Theorem 3.2 for $\zeta=\vartheta$, we get Theorem 3.1.

Theorem 3.4 Assume that there are two positive and continuous functions $\mathcal{P}$ and $\mathcal{Q}$ defined on $[0, \infty)$ with the assumption that $\mathcal{R}:[0, \infty) \rightarrow \mathbb{R}$ is positive and continuous. Then for all $\varsigma>0$, we have the inequality

$$
\begin{gathered}
{ }^{\varphi} \mathcal{K}_{0^{+}}^{\zeta, \sigma}\left[\mathcal{R}(\varsigma) \mathcal{P}^{\beta}(\varsigma)\right]^{\varphi} \mathcal{K}_{0^{+}}^{\zeta, \sigma}\left[\mathcal{R}(\varsigma) \mathcal{Q}^{\rho}(\varsigma) \mathcal{P}^{\eta}(\varsigma)\right] \\
\geq{ }^{\varphi} \mathcal{K}_{0^{+}}^{\zeta, \sigma}\left[\mathcal{R}(\varsigma) \mathcal{P}^{\eta}(\varsigma)\right]^{\varphi} \mathcal{K}_{0^{+}}^{\zeta, \sigma}\left[\mathcal{R}(\varsigma) \mathcal{Q}^{\rho}(\varsigma) \mathcal{P}^{\beta}(\varsigma)\right],
\end{gathered}
$$

where $\beta \geq \eta>0, \rho>0, \sigma \in(0,1], \zeta \in \mathcal{C}, \mathfrak{R}(\zeta)>0$. 
Proof Since $\mathcal{P}$ and $\mathcal{Q}$ are positive and continuous functions defined on $[0, \infty)$ such that a decreasing function $\mathcal{P}$ and an increasing function $\mathcal{Q}$ defined on $[0, \infty)$, hence for all $\rho>0$, $\beta \geq \eta>0, y_{1}, z_{1} \in(0, \varsigma), \varsigma>0$, we have

$$
\left(\mathcal{Q}^{\rho}\left(z_{1}\right)-\mathcal{Q}^{\rho}\left(y_{1}\right)\right)\left(\mathcal{P}^{\beta-\eta}\left(y_{1}\right)-\mathcal{P}^{\beta-\eta}\left(z_{1}\right)\right) \geq 0
$$

Inequality (3.6) leads to

$$
\mathcal{Q}^{\rho}\left(z_{1}\right) \mathcal{P}^{\beta-\eta}\left(y_{1}\right)+\mathcal{Q}^{\rho}\left(y_{1}\right) \mathcal{P}^{\beta-\eta}\left(z_{1}\right) \geq \mathcal{Q}^{\rho}\left(z_{1}\right) \mathcal{P}^{\beta-\eta}\left(z_{1}\right)+\mathcal{Q}^{\rho}\left(y_{1}\right) \mathcal{P}^{\rho}\left(z_{1}\right) \mathcal{P}^{\beta-\eta}\left(y_{1}\right) .
$$

Multiplying both sides of (3.7) by $\frac{1}{\sigma^{\zeta} \Gamma(\zeta)} \frac{\exp \left[\frac{\sigma-1}{\sigma}\left(\varphi(\varsigma)-\varphi\left(y_{1}\right)\right)\right]}{\left(\varphi(\varsigma)-\varphi\left(y_{1}\right)\right)^{1-\zeta}} \varphi^{\prime}\left(y_{1}\right) \mathcal{R}\left(y_{1}\right) \mathcal{P}^{\eta}\left(y_{1}\right)$ and then integrating the obtained inequality with respect to $y_{1}$ over $(0, \varsigma)$, we get

$$
\begin{aligned}
& \frac{\mathcal{Q}^{\rho}\left(z_{1}\right)}{\sigma^{\zeta} \Gamma(\zeta)} \int_{0}^{\varsigma} \frac{\exp \left[\frac{\sigma-1}{\sigma}\left(\varphi(\varsigma)-\varphi\left(y_{1}\right)\right)\right]}{\left(\varphi(\varsigma)-\varphi\left(y_{1}\right)\right)^{1-\zeta}} \varphi^{\prime}\left(y_{1}\right) \mathcal{R}\left(y_{1}\right) \mathcal{P}^{\eta}\left(y_{1}\right) \mathcal{P}^{\beta-\eta}\left(y_{1}\right) d y_{1} \\
& \quad+\frac{\mathcal{P}^{\beta-\eta}\left(z_{1}\right)}{\sigma^{\zeta} \Gamma(\zeta)} \int_{0}^{\varsigma} \frac{\exp \left[\frac{\sigma-1}{\sigma}\left(\varphi(\varsigma)-\varphi\left(y_{1}\right)\right)\right]}{\left(\varphi(\varsigma)-\varphi\left(y_{1}\right)\right)^{1-\zeta}} \varphi^{\prime}\left(y_{1}\right) \mathcal{R}\left(y_{1}\right) \mathcal{P}^{\eta}\left(y_{1}\right) \mathcal{Q}^{\rho} d y_{1} \\
& \geq \frac{\mathcal{Q}^{\rho}\left(z_{1}\right) \mathcal{P}^{\beta-\eta}\left(z_{1}\right)}{\sigma^{\zeta} \Gamma(\zeta)} \int_{0}^{\varsigma} \frac{\exp \left[\frac{\sigma-1}{\sigma}\left(\varphi(\varsigma)-\varphi\left(y_{1}\right)\right)\right]}{\left(\varphi(\varsigma)-\varphi\left(y_{1}\right)\right)^{1-\zeta}} \varphi^{\prime}\left(y_{1}\right) \mathcal{R}\left(y_{1}\right) \mathcal{P}^{\eta}\left(y_{1}\right)\left(y_{1}\right) d y_{1} \\
& \quad+\frac{1}{\sigma^{\zeta} \Gamma(\zeta)} \int_{0}^{\varsigma} \frac{\exp \left[\frac{\sigma-1}{\sigma}\left(\varphi(\varsigma)-\varphi\left(y_{1}\right)\right)\right]}{\left(\varphi(\varsigma)-\varphi\left(y_{1}\right)\right)^{1-\zeta}} \varphi^{\prime}\left(y_{1}\right) \mathcal{R}\left(y_{1}\right) \mathcal{P}^{\eta}\left(y_{1}\right) \mathcal{Q}^{\rho}\left(y_{1}\right) \mathcal{P}^{\beta-\eta}\left(y_{1}\right) d y_{1} .
\end{aligned}
$$

From Definition 2.3 we clearly see that

$$
\begin{aligned}
& \mathcal{Q}^{\rho}\left(z_{1}\right)^{\varphi} \mathcal{K}_{0^{+}}^{\zeta, \sigma}\left[\mathcal{R}(\varsigma) \mathcal{P}^{\beta}(\varsigma)\right]+\mathcal{P}^{\beta-\eta}\left(z_{1}\right)^{\varphi} \mathcal{K}_{0^{+}}^{\zeta, \sigma}\left[\mathcal{R}(\varsigma) \mathcal{Q}^{\rho}(\varsigma) \mathcal{P}^{\eta}(\varsigma)\right] \\
& \geq \mathcal{Q}^{\rho}\left(z_{1}\right) \mathcal{P}^{\beta-\eta}\left(z_{1}\right)^{\varphi} \mathcal{K}_{0^{+}}^{\zeta, \sigma}\left[\mathcal{R}(\varsigma) \mathcal{P}^{\eta}(\varsigma)\right]+{ }^{\varphi} \mathcal{K}_{0^{+}}^{\zeta, \sigma}\left[\mathcal{R}(\varsigma) \mathcal{Q}^{\rho}(\varsigma) \mathcal{P}^{\beta}(\varsigma)\right] .
\end{aligned}
$$

Further, if we multiply both sides of (3.8) by $\frac{1}{\sigma^{\zeta} \Gamma(\zeta)} \frac{\exp \left[\frac{\sigma-1}{\sigma}\left(\varphi(\varsigma)-\varphi\left(z_{1}\right)\right)\right]}{\left(\varphi(\zeta)-\varphi\left(z_{1}\right)\right)^{1-\zeta}} \varphi^{\prime}\left(z_{1}\right) \mathcal{R}\left(z_{1}\right) \mathcal{P}^{\eta}\left(z_{1}\right)$ and integrate the obtained inequality with respect to $z_{1}$ over $(0, \varsigma)$, and then employ (2.3), we obtain

$$
\begin{aligned}
&{ }^{\varphi} \mathcal{K}_{0^{+}}^{\zeta, \sigma} {\left[\mathcal{R}(\varsigma) \mathcal{Q}^{\rho}(\varsigma) \mathcal{P}^{\eta}(\varsigma)\right]^{\varphi} \mathcal{K}_{0^{+}}^{\zeta, \sigma}\left[\mathcal{R}(\varsigma) \mathcal{P}^{\beta}(\varsigma)\right] } \\
&{ }^{\varphi}{ }^{\varphi} \mathcal{K}_{0^{+}}^{\zeta, \sigma}\left[\mathcal{R}(\varsigma) \mathcal{P}^{\beta}(\varsigma)\right]^{\varphi} \mathcal{K}_{0^{+}}^{\zeta, \sigma}\left[\mathcal{R}(\varsigma) \mathcal{Q}^{\rho}(\varsigma) \mathcal{P}^{\eta}(\varsigma)\right] \\
& \geq{ }^{\varphi} \mathcal{K}_{0^{+}}^{\zeta, \sigma}\left[\mathcal{R}(\varsigma) \mathcal{Q}^{\rho}(\varsigma) \mathcal{P}^{\beta}(\varsigma)\right]^{\varphi} \mathcal{K}_{0^{+}}^{\zeta, \sigma}\left[\mathcal{R}(\varsigma) \mathcal{P}^{\eta}(\varsigma)\right] \\
&{ }^{\varphi}{ }^{\varphi} \mathcal{K}_{0^{+}}^{\zeta, \sigma}\left[\mathcal{R}(\varsigma) \mathcal{P}^{\eta}(\varsigma)\right]^{\varphi} \mathcal{K}_{0^{+}}^{\zeta, \sigma}\left[\mathcal{R}(\varsigma) \mathcal{Q}^{\rho}(\varsigma) \mathcal{P}^{\beta}(\varsigma)\right],
\end{aligned}
$$

which gives the desired inequality (3.5).

Theorem 3.5 Assume that there are two positive and continuous functions $\mathcal{P}$ and $\mathcal{Q}$ defined on $[0, \infty)$, function $\mathcal{P}$ is decreasing and function $\mathcal{Q}$ is an increasing $[0, \infty)$, with the assumption that the function $\mathcal{R}:[0, \infty) \rightarrow \mathbb{R}$ is positive and continuous. Then for all $\varsigma>0$, one has

$$
\begin{aligned}
{ }^{\varphi} \mathcal{K}_{0^{+}}^{\zeta, \sigma} & {\left[\mathcal{R}(\varsigma) \mathcal{P}^{\beta}(\varsigma)\right]^{\varphi} \mathcal{K}_{0^{+}}^{\vartheta, \sigma}\left[\mathcal{R}(\varsigma) \mathcal{Q}^{\rho}(\varsigma) \mathcal{P}^{\eta}(\varsigma)\right] } \\
& +{ }^{\varphi} \mathcal{K}_{0^{+}}^{\vartheta, \sigma}\left[\mathcal{R}(\varsigma) \mathcal{P}^{\beta}(\varsigma)\right]^{\varphi} \mathcal{K}_{0^{+}}^{\zeta, \sigma}\left[\mathcal{R}(\varsigma) \mathcal{Q}^{\rho}(\varsigma) \mathcal{P}^{\eta}(\varsigma)\right]
\end{aligned}
$$




$$
\begin{aligned}
\geq & { }^{\varphi} \mathcal{K}_{0^{+}}^{\zeta, \sigma}\left[\mathcal{R}(\varsigma) \mathcal{P}^{\eta}(\varsigma)\right]^{\varphi} \mathcal{K}_{0^{+}}^{\vartheta, \sigma}\left[\mathcal{R}(\varsigma) \mathcal{Q}^{\rho}(\varsigma) \mathcal{P}^{\beta}(\varsigma)\right] \\
& +{ }^{\varphi} \mathcal{K}_{0^{+}}^{\vartheta, \sigma}\left[\mathcal{R}(\varsigma) \mathcal{P}^{\eta}(\varsigma)\right]^{\varphi} \mathcal{K}_{0^{+}}^{\zeta, \sigma}\left[\mathcal{R}(\varsigma) \mathcal{Q}^{\rho}(\varsigma) \mathcal{P}^{\beta}(\varsigma)\right]
\end{aligned}
$$

where $\beta \geq \eta>0, \rho>0, \sigma \in(0,1], \zeta \in \mathcal{C}, \mathfrak{R}(\zeta)>0$.

Proof Multiplying both sides of (3.8) by $\frac{1}{\sigma^{\vartheta} \Gamma(\vartheta)} \frac{\exp \left[\frac{\sigma-1}{\sigma}\left(\varphi(\varsigma)-\varphi\left(z_{1}\right)\right)\right]}{\left(\varphi(\varsigma)-\varphi\left(z_{1}\right)\right)^{1-\vartheta}} \varphi^{\prime}\left(z_{1}\right) \mathcal{R}\left(z_{1}\right) \mathcal{P}^{\eta}\left(z_{1}\right)$, where $\vartheta, \eta>0$ and $z_{1} \in(0, \varsigma), \varsigma>0$, and then integrating the previous inequality with respect to $z_{1}$ over $(0, \varsigma)$, we have

$$
\begin{aligned}
\frac{\varphi \mathcal{K}_{0^{+}}^{\zeta, \sigma}}{\left.\sigma^{\vartheta} \Gamma(\varsigma) \mathcal{P}^{\beta}(\varsigma)\right]} \int_{0}^{\varsigma} \frac{\exp \left[\frac{\sigma-1}{\sigma}\left(\varphi(\varsigma)-\varphi\left(z_{1}\right)\right)\right]}{\left(\varphi(\varsigma)-\varphi\left(z_{1}\right)\right)^{1-\vartheta}} \varphi^{\prime}\left(z_{1}\right) \mathcal{R}\left(z_{1}\right) \mathcal{P}^{\eta}\left(z_{1}\right) \mathcal{Q}^{\rho}\left(z_{1}\right) d z_{1} \\
\quad+\frac{\varphi \mathcal{K}_{0^{+}}^{\zeta, \sigma}\left[\mathcal{R}(\varsigma) \mathcal{Q}^{\rho}(\varsigma) \mathcal{P}^{\eta}(\varsigma)\right]}{\sigma^{\vartheta} \Gamma(\vartheta)} \\
\quad \times \int_{0}^{\varsigma} \frac{\exp \left[\frac{\sigma-1}{\sigma}\left(\varphi(\varsigma)-\varphi\left(z_{1}\right)\right)\right]}{\left(\varphi(\varsigma)-\varphi\left(z_{1}\right)\right)^{1-\vartheta}} \varphi^{\prime}\left(z_{1}\right) \mathcal{R}\left(z_{1}\right) \mathcal{P}^{\eta}\left(z_{1}\right) \mathcal{P}^{\beta-\eta}\left(z_{1}\right) d z_{1} \\
\geq \frac{\varphi \mathcal{K}_{0^{+}}^{\zeta, \sigma}\left[\mathcal{R}(\varsigma) \mathcal{P}^{\rho}(\varsigma)\right]}{\sigma^{\vartheta} \Gamma(\vartheta)} \\
\quad \times \int_{0}^{\varsigma} \frac{\exp \left[\frac{\sigma-1}{\sigma}\left(\varphi(\varsigma)-\varphi\left(z_{1}\right)\right)\right]}{\left(\varphi(\varsigma)-\varphi\left(z_{1}\right)\right)^{1-\vartheta}} \varphi^{\prime}\left(z_{1}\right) \mathcal{R}\left(z_{1}\right) \mathcal{P}^{\eta}\left(z_{1}\right) \mathcal{Q}^{\rho}\left(z_{1}\right) \mathcal{P}^{\beta-\eta}\left(z_{1}\right) d z_{1} \\
\quad+\frac{{ }^{\varphi} \mathcal{K}_{0^{+}}^{\zeta, \sigma}\left[\mathcal{R}(\varsigma) \mathcal{Q}^{\rho}(\varsigma) \mathcal{P}^{\beta}(\varsigma)\right]}{\sigma^{\vartheta} \Gamma(\vartheta)} \int_{0}^{\varsigma} \frac{\exp \left[\frac{\sigma-1}{\sigma}\left(\varphi(\varsigma)-\varphi\left(z_{1}\right)\right)\right]}{\left(\varphi(\varsigma)-\varphi\left(z_{1}\right)\right)^{1-\vartheta}} \varphi^{\prime}\left(z_{1}\right) \mathcal{R}\left(z_{1}\right) \mathcal{P}^{\eta}\left(z_{1}\right) d z_{1} .
\end{aligned}
$$

From (2.3) we clearly see that the above inequality can be rewritten as

$$
\begin{aligned}
{ }^{\varphi} \mathcal{K}_{0^{+}}^{\zeta, \sigma} & {\left[\mathcal{R}(\varsigma) \mathcal{P}^{\beta}(\varsigma)\right]^{\varphi} \mathcal{K}_{0^{+}}^{\vartheta, \sigma}\left[\mathcal{R}(\varsigma) \mathcal{Q}^{\rho}(\varsigma) \mathcal{P}^{\eta}(\varsigma)\right] } \\
& { }^{\varphi} \mathcal{K}_{0^{+}}^{\zeta, \sigma}\left[\mathcal{R}(\varsigma) \mathcal{Q}^{\rho}(\varsigma) \mathcal{P}^{\eta}(\varsigma)\right]^{\varphi} \mathcal{K}_{0^{+}}^{\vartheta, \sigma}\left[\mathcal{R}(\varsigma) \mathcal{P}^{\beta}(\varsigma)\right] \\
\geq{ }^{\varphi} & \mathcal{K}_{0^{+}}^{\zeta, \sigma}\left[\mathcal{R}(\varsigma) \mathcal{P}^{\eta}(\varsigma)\right]^{\varphi} \mathcal{K}_{0^{+}}^{\vartheta, \sigma}\left[\mathcal{R}(\varsigma) \mathcal{Q}^{\rho}(\varsigma) \mathcal{P}^{\beta}(\varsigma)\right] \\
& +{ }^{\varphi} \mathcal{K}_{0^{+}}^{\zeta, \sigma}\left[\mathcal{R}(\varsigma) \mathcal{Q}^{\rho}(\varsigma) \mathcal{P}^{\beta}(\varsigma)\right]^{\varphi} \mathcal{K}_{0^{+}}^{\vartheta, \sigma}\left[\mathcal{R}(\varsigma) \mathcal{P}^{\eta}(\varsigma)\right],
\end{aligned}
$$

which is the desired inequality (3.9).

Remark 3.6 Applying Theorem 3.5 for $\zeta=\vartheta$, we get Theorem 3.4.

Theorem 3.7 Assume that there are two functions $\mathcal{P}_{s}(s=1,2, \ldots, n)$ and $\mathcal{Q}$ which are positive and continuous, defined on $[0, \infty)$, with the assumption that the function $\mathcal{R}:[0, \infty) \rightarrow$ $\mathbb{R}$ is positive and continuous. Then for all $\varsigma>0$, we have

$$
\begin{aligned}
& { }^{\varphi} \mathcal{K}_{0^{+}}^{\zeta, \sigma}\left[\mathcal{R}(\varsigma) \mathcal{P}_{p}^{\beta+\rho}(\varsigma) \prod_{s \neq p}^{n} \mathcal{P}_{s}^{\eta_{s}}(\varsigma)\right]{ }^{\varphi} \mathcal{K}_{0^{+}}^{\zeta, \sigma}\left[\mathcal{R}(\varsigma) \mathcal{Q}^{\rho}(\varsigma) \prod_{s=1}^{n} \mathcal{P}^{\eta_{s}}(\varsigma)\right] \\
& \geq{ }^{\varphi} \mathcal{K}_{0^{+}}^{\zeta, \sigma}\left[\mathcal{R}(\varsigma) \mathcal{P}_{p}^{\rho}(\varsigma) \prod_{s=1}^{n} \mathcal{P}^{\eta_{s}}(\varsigma)\right] \varphi \mathcal{K}_{0^{+}}^{\zeta, \sigma}\left[\mathcal{R}(\varsigma) \mathcal{Q}^{\rho}(\varsigma) \mathcal{U}_{p}^{\beta}(\varsigma) \prod_{s \neq p}^{n} \mathcal{P}_{s}^{\eta_{s}}(\varsigma)\right],
\end{aligned}
$$

where $\beta \geq \eta_{p}>0, \rho>0(p=1,2, \ldots, n), \sigma \in(0,1], \zeta \in \mathcal{C}, \mathfrak{R}(\zeta)>0$. 
Proof Since $\mathcal{P}_{s}$ and $\mathcal{Q}$ are positive and continuous functions on $[0, \infty)$, for all $y_{1}, z_{1} \in(0, \varsigma)$, $\varsigma>0$ and for any $\rho>0, \beta \leq \eta_{p}>0$, we have

$$
\left(\mathcal{Q}^{\rho}\left(z_{1}\right) \mathcal{P}_{p}^{\rho}\left(y_{1}\right)-\mathcal{Q}^{\rho}\left(y_{1}\right) \mathcal{P}^{\rho}\left(z_{1}\right)\right)\left(\mathcal{P}_{p}^{\beta-\eta_{p}}\left(y_{1}\right)-\mathcal{P}_{p}^{\beta-\eta_{p}}\left(z_{1}\right)\right) \geq 0
$$

It follows from (3.11) that

$$
\begin{aligned}
& \mathcal{Q}^{\rho}\left(z_{1}\right) \mathcal{P}_{p}^{\rho+\beta-\eta_{p}}\left(y_{1}\right)+\mathcal{Q}^{\rho}\left(y_{1}\right) \mathcal{P}_{p}^{\rho+\beta-\eta_{p}}\left(z_{1}\right) \\
& \quad \geq \mathcal{Q}^{\rho}\left(z_{1}\right) \mathcal{P}_{p}^{\rho}\left(y_{1}\right) \mathcal{P}_{p}^{\beta-\eta_{p}}\left(z_{1}\right)+\mathcal{Q}^{\rho}\left(y_{1}\right) \mathcal{P}_{p}^{\rho}\left(z_{1}\right) \mathcal{P}_{p}^{\beta-\eta_{p}}\left(y_{1}\right) .
\end{aligned}
$$

Multiplying both sides of (3.12) by $\frac{1}{\sigma^{\zeta} \Gamma(\zeta)} \frac{\exp \left[\frac{\sigma-1}{\sigma}\left(\varphi(\varsigma)-\varphi\left(y_{1}\right)\right)\right]}{\left.\left(\varphi(\varsigma)-\varphi\left(y_{1}\right)\right)\right)^{1-\zeta}} \varphi^{\prime}\left(y_{1}\right) \mathcal{R}\left(y_{1}\right) \prod_{s=1}^{n} \mathcal{P}_{s}^{\eta_{s}}\left(y_{1}\right)\left(\eta_{s}>0\right.$, $s=1,2, \ldots, n$ and $\left.y_{1} \in(0, \varsigma)\right)$ and then integrating the obtained inequality with respect to $y_{1}$ over $(0, \varsigma)$, we have

$$
\begin{aligned}
& \frac{\mathcal{Q}^{\rho}\left(z_{1}\right)}{\sigma^{\zeta} \Gamma(\zeta)} \int_{0}^{\varsigma} \frac{\exp \left[\frac{\sigma-1}{\sigma}\left(\varphi(\varsigma)-\varphi\left(y_{1}\right)\right)\right]}{\left(\varphi(\varsigma)-\varphi\left(y_{1}\right)\right)^{1-\zeta}} \varphi^{\prime}\left(y_{1}\right) \mathcal{R}\left(y_{1}\right) \prod_{s=1}^{n} \mathcal{P}_{s}^{\eta_{s}}\left(y_{1}\right) \mathcal{P}_{p}^{\rho+\beta-\eta_{p}}\left(y_{1}\right) d y_{1} \\
& \quad+\frac{\mathcal{P}_{p}^{\rho+\beta-\eta_{p}}\left(z_{1}\right)}{\sigma^{\zeta} \Gamma(\zeta)} \int_{0}^{\varsigma} \frac{\exp \left[\frac{\sigma-1}{\sigma}\left(\varphi(\varsigma)-\varphi\left(y_{1}\right)\right)\right]}{\left(\varphi(\varsigma)-\varphi\left(y_{1}\right)\right)^{1-\zeta}} \varphi^{\prime}\left(y_{1}\right) \mathcal{R}\left(y_{1}\right) \prod_{s=1}^{n} \mathcal{P}_{s}^{\eta_{s}}\left(y_{1}\right) \mathcal{Q}^{\rho}\left(y_{1}\right) d y_{1} \\
& \geq \frac{\mathcal{Q}^{\rho}\left(z_{1}\right) \mathcal{P}_{p}^{\beta-\eta_{p}}\left(z_{1}\right)}{\sigma^{\zeta} \Gamma(\zeta)} \int_{0}^{\varsigma} \frac{\exp \left[\frac{\sigma-1}{\sigma}\left(\varphi(\varsigma)-\varphi\left(y_{1}\right)\right)\right]}{\left(\varphi(\varsigma)-\varphi\left(y_{1}\right)\right)^{1-\zeta}} \varphi^{\prime}\left(y_{1}\right) \mathcal{R}\left(y_{1}\right) \prod_{s=1}^{n} \mathcal{P}_{s}^{\eta_{s}}\left(y_{1}\right) \mathcal{P}_{p}^{\rho}\left(y_{1}\right) d y_{1} \\
& \quad+\frac{\mathcal{P}_{p}^{\rho}\left(z_{1}\right)}{\sigma^{\zeta} \Gamma(\zeta)} \int_{0}^{\varsigma} \frac{\exp \left[\frac{\sigma-1}{\sigma}\left(\varphi(\varsigma)-\varphi\left(y_{1}\right)\right)\right]}{\left(\varphi(\varsigma)-\varphi\left(y_{1}\right)\right)^{1-\zeta}} \varphi^{\prime}\left(y_{1}\right) \mathcal{R}\left(y_{1}\right) \\
& \quad \times \prod_{s=1}^{n} \mathcal{P}_{s}^{\eta_{s}}\left(y_{1}\right) \mathcal{Q}^{\rho}\left(y_{1}\right) \mathcal{P}_{p}^{\beta-\eta_{p}}\left(y_{1}\right) d y_{1} .
\end{aligned}
$$

In view of Definition 2.3, we have

$$
\begin{aligned}
\mathcal{Q}^{\rho}\left(z_{1}\right)^{\varphi} \mathcal{K}_{0^{+}}^{\zeta, \sigma}\left[\mathcal{R}(\varsigma) \mathcal{P}_{p}^{\beta+\rho}(\varsigma) \prod_{s \neq p}^{n} \mathcal{P}_{s}^{\eta_{s}}(\varsigma)\right] \\
+\mathcal{P}_{p}^{\rho+\beta-\eta}\left(z_{1}\right) \mathcal{H}_{1, \zeta}^{\zeta, \rho}\left[\mathcal{R}(\varsigma) \mathcal{Q}^{\rho}(\varsigma) \mathcal{P}_{p}^{\eta_{p}}(\varsigma) \prod_{s \neq p}^{n} \mathcal{P}_{s}^{\eta_{s}}(\varsigma) \prod_{s=1}^{n} \mathcal{P}_{s}^{\eta_{s}}(\varsigma)\right] \\
\geq \mathcal{Q}^{\rho}\left(z_{1}\right) \mathcal{P}_{p}^{\beta-\eta_{p}}\left(z_{1}\right)^{\varphi} \mathcal{K}_{0^{+}}^{\zeta, \sigma}\left[\mathcal{R}(\varsigma) \mathcal{P}^{\rho+\eta}\left(y_{1}\right) \prod_{s \neq p}^{n} \mathcal{P}_{s}^{\eta_{s}}(\varsigma)\right] \\
\quad+\mathcal{P}_{p}^{\rho}\left(z_{1}\right)^{\varphi} \mathcal{K}_{0^{+}}^{\zeta, \sigma}\left[\mathcal{R}(\varsigma) \mathcal{Q}^{\rho}(\varsigma) \mathcal{P}^{\beta}(\varsigma) \prod_{s=1}^{n} \mathcal{P}_{s}^{\eta_{s}}(\varsigma)\right] .
\end{aligned}
$$

Again, if we multiply both sides of (3.13) by $\frac{1}{\sigma \zeta \Gamma(\zeta)} \frac{\exp \left[\frac{\sigma-1}{\sigma}\left(\varphi(\varsigma)-\varphi\left(z_{1}\right)\right)\right]}{\left(\varphi(\varsigma)-\varphi\left(z_{1}\right)\right)^{1-\zeta}} \varphi^{\prime}\left(z_{1}\right) \mathcal{R}\left(z_{1}\right) \prod_{s=1}^{n} \mathcal{P}_{s}^{\eta_{s}}\left(z_{1}\right)$ $\left(\eta_{s}>0, s=1,2, \ldots, n\right.$ and $\left.z_{1} \in(0, \varsigma)\right)$ and integrate the obtained inequality with respect to $z_{1}$ over $(0, \varsigma)$, then, by using $(2.3)$, we obtain

$$
{ }^{\varphi} \mathcal{K}_{0^{+}}^{\zeta, \sigma}\left[\mathcal{R}(\varsigma) \mathcal{P}_{p}^{\rho+\beta}(\varsigma) \prod_{s \neq p}^{n} \mathcal{P}_{s}^{\eta_{s}}(\varsigma)\right] \varphi \mathcal{K}_{0^{+}}^{\zeta, \sigma}\left[\mathcal{R}(\varsigma) \mathcal{Q}^{\rho}(\varsigma) \prod_{s=1}^{n} \mathcal{P}_{s}^{\eta_{s}}(\varsigma)\right]
$$




$$
\begin{aligned}
&+{ }^{\varphi} \mathcal{K}_{0^{+}}^{\zeta, \sigma}\left[\mathcal{R}(\varsigma) \mathcal{Q}^{\rho}(\varsigma) \prod_{s=1}^{n} \mathcal{P}_{s}^{\eta_{s}}(\varsigma)\right]{ }^{\varphi} \mathcal{K}_{0^{+}}^{\zeta, \sigma}\left[\mathcal{R}(\varsigma) \mathcal{P}_{p}^{\rho+\beta}(\varsigma) \prod_{s \neq p}^{n} \mathcal{P}_{s}^{\eta_{s}}(\varsigma)\right] \\
& \geq{ }^{\varphi} \mathcal{K}_{0^{+}}^{\zeta, \sigma}\left[\mathcal{R}(\varsigma) \mathcal{P}_{p}^{\rho}(\varsigma) \prod_{s=1}^{n} \mathcal{P}_{s}^{\eta_{s}}(\varsigma)\right] \varphi \mathcal{K}_{0^{+}}^{\zeta, \sigma}\left[\mathcal{R}(\varsigma) \mathcal{Q}^{\rho}(\varsigma) \mathcal{P}_{p}^{\beta}(\varsigma) \prod_{s \neq p}^{n} \mathcal{P}_{s}^{\eta_{s}}(\varsigma)\right] \\
& \quad+{ }^{\varphi} \mathcal{K}_{0^{+}}^{\zeta, \sigma}\left[\mathcal{R}(\varsigma) \mathcal{Q}^{\rho}(\varsigma) \mathcal{P}_{p}^{\beta}(\varsigma) \prod_{s \neq p}^{n} \mathcal{P}_{s}^{\eta_{s}}(\varsigma)\right]{ }^{\varphi} \mathcal{K}_{0^{+}}^{\zeta, \sigma}\left[\mathcal{R}(\varsigma) \mathcal{P}_{p}^{\rho}(\varsigma) \prod_{s=1}^{n} \mathcal{P}_{s}^{\eta_{s}}(\varsigma)\right],
\end{aligned}
$$

which is the desired inequality (3.10).

Theorem 3.8 Assume that there are two functions $\mathcal{P}_{s}(s=1,2, \ldots, n)$ and $\mathcal{Q}$ which are positive and continuous, defined on $[0, \infty)$, with the assumption that the function $\mathcal{R}:[0, \infty) \rightarrow$ $\mathbb{R}$ is positive and continuous. Then for all $\varsigma>0$, we have the inequality

$$
\begin{aligned}
&{ }^{\varphi} \mathcal{K}_{0^{+}}^{\zeta, \sigma} {\left[\mathcal{R}(\varsigma) \mathcal{P}_{p}^{\rho+\beta}(\varsigma) \prod_{s \neq p}^{n} \mathcal{P}_{s}^{\eta_{s}}(\varsigma)\right]{ }^{\varphi} \mathcal{K}_{0^{+}}^{\vartheta, \sigma}\left[\mathcal{R}(\varsigma) \mathcal{Q}^{\rho}(\varsigma) \prod_{s=1}^{n} \mathcal{P}_{p}^{\eta_{s}}(\varsigma)\right] } \\
&+{ }^{\varphi} \mathcal{K}_{0^{+}}^{\vartheta, \sigma}\left[\mathcal{R}(\varsigma) \mathcal{P}_{p}^{\rho+\beta}(\varsigma) \prod_{s \neq p}^{n} \mathcal{P}_{s}^{\eta_{s}}(\varsigma)\right]{ }^{\varphi} \mathcal{K}_{0^{+}}^{\zeta, \sigma}\left[\mathcal{R}(\varsigma) \mathcal{Q}^{\rho}(\varsigma) \prod_{s=1}^{n} \mathcal{P}_{p}^{\eta_{s}}(\varsigma)\right] \\
& \geq{ }^{\varphi} \mathcal{K}_{0^{+}}^{\zeta, \sigma}\left[\mathcal{R}(\varsigma) \mathcal{P}_{p}^{\rho}(\varsigma) \prod_{s=1}^{n} \mathcal{P}_{p}^{\eta_{s}}(\varsigma)\right]{ }^{\varphi} \mathcal{K}_{0^{+}}^{\vartheta, \sigma}\left[\mathcal{R}(\varsigma) \mathcal{Q}^{\rho}(\varsigma) \mathcal{P}_{p}^{\beta}(\varsigma) \prod_{s \neq p}^{n} \mathcal{P}_{s}^{\eta_{s}}(\varsigma)\right] \\
&+{ }^{\varphi} \mathcal{K}_{0^{+}}^{\zeta, \sigma}\left[\mathcal{R}(\varsigma) \mathcal{P}_{p}^{\rho}(\varsigma) \prod_{s=1}^{n} \mathcal{P}_{p}^{\eta_{s}}(\varsigma)\right]{ }^{\varphi} \mathcal{K}_{0^{+}}^{\vartheta, \sigma}\left[\mathcal{R}(\varsigma) \mathcal{Q}^{\rho}(\varsigma) \mathcal{P}_{p}^{\beta}(\varsigma) \prod_{s \neq p}^{n} \mathcal{P}_{s}^{\eta_{s}}(\varsigma)\right],
\end{aligned}
$$

where $\beta \geq \eta_{p}>0, \rho>0(p=1,2, \ldots, n), \sigma \in(0,1], \zeta \in \mathcal{C}, \mathfrak{R}(\zeta)>0$.

Proof Multiplying both sides of (3.13) by $\frac{1}{\sigma^{\vartheta} \Gamma(\vartheta)} \frac{\exp \left[\frac{\sigma-1}{\sigma}\left(\varphi(\varsigma)-\varphi\left(z_{1}\right)\right)\right]}{\left(\varphi(\varsigma)-\varphi\left(z_{1}\right)\right)^{1-\vartheta}} \varphi^{\prime}\left(z_{1}\right) \mathcal{R}\left(z_{1}\right) \times$ $\prod_{s=1}^{n} \mathcal{P}^{\eta}(\varsigma)\left(z_{1}\right)$, where $\vartheta, \eta_{s}>0(s=1,2, \ldots, n), z_{1} \in(0, \varsigma), \varsigma>0$ and integrating the obtained inequality with respect to $z_{1}$ over $(0, \varsigma)$, we have

$$
\begin{aligned}
\frac{\varphi \mathcal{K}_{0^{+}}^{\zeta, \sigma}\left[\mathcal{R}(\varsigma) \mathcal{P}_{p}^{\rho+\beta}(\varsigma) \prod_{s \neq p}^{n} \mathcal{P}_{s}^{\eta_{s}}(\varsigma)\right]}{\sigma^{\vartheta} \Gamma(\vartheta)} \\
\quad \times \int_{0}^{\varsigma} \frac{\exp \left[\frac{\sigma-1}{\sigma}\left(\varphi(\varsigma)-\varphi\left(z_{1}\right)\right)\right]}{\left(\varphi(\varsigma)-\varphi\left(z_{1}\right)\right)^{1-\vartheta}} \varphi^{\prime}\left(z_{1}\right) \mathcal{R}\left(z_{1}\right) \prod_{s=1}^{n} \mathcal{P}_{s}^{\eta}\left(z_{1}\right) \mathcal{Q}^{\rho}\left(z_{1}\right) d z_{1} \\
\quad+\frac{\varphi \mathcal{K}_{0^{+}}^{\zeta, \sigma}\left[\mathcal{R}(\varsigma) \mathcal{Q}^{\rho}(\varsigma) \prod_{s=1}^{n} \mathcal{P}_{s}^{\eta_{s}}(\varsigma)\right]}{\sigma^{\vartheta} \Gamma(\vartheta)} \\
\quad \times \int_{0}^{\varsigma} \frac{\exp \left[\frac{\sigma-1}{\sigma}\left(\varphi(\varsigma)-\varphi\left(z_{1}\right)\right)\right]}{\left(\varphi(\varsigma)-\varphi\left(z_{1}\right)\right)^{1-\vartheta}} \varphi^{\prime}\left(z_{1}\right) \mathcal{R}\left(z_{1}\right) \prod_{s=1}^{n} \mathcal{P}_{s}^{\eta}\left(z_{1}\right) \mathcal{P}_{p}^{\rho+\beta-\eta_{p}}\left(z_{1}\right) d z_{1} \\
\geq \frac{\varphi \mathcal{K}_{0^{+}}^{\zeta, \sigma}\left[\mathcal{R}(\varsigma) \mathcal{P}^{\rho}(\varsigma) \prod_{s=1}^{n} \mathcal{P}_{s}^{\eta_{s}}(\varsigma)\right]}{\sigma^{\vartheta} \Gamma(\vartheta)} \\
\quad \times \int_{0}^{\varsigma} \frac{\exp \left[\frac{\sigma-1}{\sigma}\left(\varphi(\varsigma)-\varphi\left(z_{1}\right)\right)\right]}{\left(\varphi(\varsigma)-\varphi\left(z_{1}\right)\right)^{1-\vartheta}} \varphi^{\prime}\left(z_{1}\right) \mathcal{R}\left(z_{1}\right) \prod_{s=1}^{n} \mathcal{P}_{s}^{\eta}\left(z_{1}\right) \mathcal{Q}^{\rho}\left(z_{1}\right) \mathcal{P}_{p}^{\beta-\eta_{p}}\left(z_{1}\right) d z_{1}
\end{aligned}
$$




$$
\begin{aligned}
& +\frac{\varphi \mathcal{K}_{0^{+}}^{\zeta, \sigma}\left[\mathcal{R}(\varsigma) \mathcal{Q}^{\rho}(\varsigma) \mathcal{P}_{p}^{\rho+\beta}(\varsigma) \prod_{s \neq p}^{n} \mathcal{P}_{s}^{\eta_{s}}(\varsigma)\right]}{\sigma^{\vartheta} \Gamma(\vartheta)} \\
& \times \int_{0}^{\varsigma} \frac{\exp \left[\frac{\sigma-1}{\sigma}\left(\varphi(\varsigma)-\varphi\left(z_{1}\right)\right)\right]}{\left(\varphi(\varsigma)-\varphi\left(z_{1}\right)\right)^{1-\vartheta}} \varphi^{\prime}\left(z_{1}\right) \mathcal{R}\left(z_{1}\right) \prod_{s=1}^{n} \mathcal{P}_{s}^{\eta}\left(z_{1}\right) \mathcal{P}_{p}^{\rho}\left(z_{1}\right) d z_{1} .
\end{aligned}
$$

It follows from Definition 2.3 that

$$
\begin{aligned}
&{ }^{\varphi} \mathcal{K}_{0^{+}}^{\zeta, \sigma} {\left[\mathcal{R}(\varsigma) \mathcal{P}_{p}^{\rho+\beta}(\varsigma) \prod_{s \neq p}^{n} \mathcal{P}_{s}^{\eta_{s}}(\varsigma)\right]{ }^{\varphi} \mathcal{K}_{0^{+}}^{\vartheta, \sigma}\left[\mathcal{R}(\varsigma) \mathcal{Q}^{\rho}(\varsigma) \prod_{s=1}^{n} \mathcal{P}_{p}^{\eta_{s}}(\varsigma)\right] } \\
&+{ }^{\varphi} \mathcal{K}_{0^{+}}^{\vartheta, \sigma}\left[\mathcal{R}(\varsigma) \mathcal{P}_{p}^{\rho+\beta}(\varsigma) \prod_{s \neq p}^{n} \mathcal{P}_{s}^{\eta_{s}}(\varsigma)\right] \mathcal{K}_{0^{+}}^{\zeta, \sigma}\left[\mathcal{R}(\varsigma) \mathcal{Q}^{\rho}(\varsigma) \prod_{s=1}^{n} \mathcal{P}_{p}^{\eta_{s}}(\varsigma)\right] \\
& \geq{ }^{\varphi} \mathcal{K}_{0^{+}}^{\zeta, \sigma}\left[\mathcal{R}(\varsigma) \mathcal{P}_{p}^{\rho}(\varsigma) \prod_{s=1}^{n} \mathcal{P}_{p}^{\eta_{s}}(\varsigma)\right]{ }^{\varphi} \mathcal{K}_{0^{+}}^{\vartheta, \sigma}\left[\mathcal{R}(\varsigma) \mathcal{Q}^{\rho}(\varsigma) \mathcal{P}_{p}^{\beta}(\varsigma) \prod_{s \neq p}^{n} \mathcal{P}_{s}^{\eta_{s}}(\varsigma)\right] \\
&+{ }^{\varphi} \mathcal{K}_{0^{+}}^{\zeta, \sigma}\left[\mathcal{R}(\varsigma) \mathcal{P}_{p}^{\rho}(\varsigma) \prod_{s=1}^{n} \mathcal{P}_{p}^{\eta_{s}}(\varsigma)\right]{ }^{\varphi} \mathcal{K}_{0^{+}}^{\vartheta, \sigma}\left[\mathcal{R}(\varsigma) \mathcal{Q}^{\rho}(\varsigma) \mathcal{P}_{p}^{\beta}(\varsigma) \prod_{s \neq p}^{n} \mathcal{P}_{s}^{\eta_{s}}(\varsigma)\right],
\end{aligned}
$$

which is the required inequality (3.14).

Remark 3.9 Applying Theorem 3.8 for $\zeta=\vartheta$, we get Theorem 3.7.

Theorem 3.10 Assume that there are two functions $\mathcal{P}_{s}(s=1,2, \ldots, n)$ and $\mathcal{Q}$ which are positive and continuous, defined on $[0, \infty)$, with the assumption that the function $\mathcal{R}:[0, \infty) \rightarrow \mathbb{R}$ is positive and continuous, function $\mathcal{Q}$ is increasing and function $\mathcal{P}_{s}$ $(s=1,2, \ldots, n)$ is decreasing on $[0, \infty)$. Then for all $\varsigma>0$, the following inequality holds:

$$
\begin{aligned}
& { }^{\varphi} \mathcal{K}_{0^{+}}^{\zeta, \sigma}\left[\mathcal{R}(\varsigma) \mathcal{P}_{p}^{\beta}(\varsigma) \prod_{s \neq p}^{n} \mathcal{P}_{s}^{\eta_{s}}(\varsigma)\right]{ }^{\varphi} \mathcal{K}_{0^{+}}^{\zeta, \sigma}\left[\mathcal{R}(\varsigma) \mathcal{Q}^{\rho}(\varsigma) \prod_{s=1}^{n} \mathcal{P}^{\eta_{s}}(\varsigma)\right] \\
& \geq{ }^{\varphi} \mathcal{K}_{0^{+}}^{\zeta, \sigma}\left[\mathcal{R}(\varsigma) \mathcal{Q}^{\rho}(\varsigma) \mathcal{P}_{p}^{\beta}(\varsigma) \prod_{s \neq p}^{n} \mathcal{P}_{s}^{\eta_{s}}(\varsigma)\right]{ }^{\varphi} \mathcal{K}_{0^{+}}^{\zeta, \sigma}\left[\mathcal{R}(\varsigma) \prod_{s=1}^{n} \mathcal{P}^{\eta_{s}}(\varsigma)\right],
\end{aligned}
$$

where $\beta \geq \eta_{p}>0, \rho>0(p=1,2, \ldots, n), \sigma \in(0,1], \zeta \in \mathcal{C}, \mathfrak{R}(\zeta)>0$.

Proof Utilizing hypothesis mentioned in Theorem 3.10, one obtains

$$
\left(\mathcal{Q}^{\rho}\left(z_{1}\right)-\mathcal{Q}^{\rho}\left(y_{1}\right)\right)\left(\mathcal{P}_{p}^{\beta-\eta_{p}}\left(y_{1}\right)-\mathcal{P}_{p}^{\beta-\eta_{p}}\left(z_{1}\right)\right) \geq 0,
$$

for any $y_{1}, z_{1} \in(0, \varsigma), \varsigma>0, \rho>0, \beta \geq \eta>0$, and $p=1,2, \ldots, n$.

It follows from (3.16) that

$$
\mathcal{Q}^{\rho}\left(z_{1}\right) \mathcal{P}_{p}^{\beta-\eta_{p}}\left(y_{1}\right)+\mathcal{Q}^{\rho}\left(y_{1}\right) \mathcal{P}_{p}^{\beta-\eta_{p}}\left(z_{1}\right) \geq \mathcal{Q}^{\rho}\left(z_{1}\right) \mathcal{P}_{p}^{\beta-\eta_{p}}\left(z_{1}\right)+\mathcal{Q}^{\rho}\left(y_{1}\right) \mathcal{P}_{p}^{\beta-\eta_{p}}\left(z_{1}\right)
$$


Multiplying both sides of (3.17) by $\frac{1}{\sigma^{\zeta} \Gamma(\zeta)} \frac{\exp \left[\frac{\sigma-1}{\sigma}\left(\varphi(\zeta)-\varphi\left(y_{1}\right)\right)\right]}{\left(\varphi(\zeta)-\varphi\left(y_{1}\right)\right)^{1-\zeta}} \varphi^{\prime}\left(y_{1}\right) \mathcal{R}\left(y_{1}\right) \prod_{s=1}^{n} \mathcal{P}_{s}^{\eta_{s}}\left(y_{1}\right)$ and integrating the obtained inequality with respect to $y_{1}$ over $(0, \varsigma)$, we have

$$
\begin{aligned}
& \frac{\mathcal{Q}^{\rho}\left(z_{1}\right)}{\sigma^{\zeta} \Gamma(\zeta)} \int_{0}^{\varsigma} \frac{\exp \left[\frac{\sigma-1}{\sigma}\left(\varphi(\varsigma)-\varphi\left(y_{1}\right)\right)\right]}{\left(\varphi(\varsigma)-\varphi\left(y_{1}\right)\right)^{1-\zeta}} \varphi^{\prime}\left(y_{1}\right) \mathcal{R}\left(y_{1}\right) \prod_{s=1}^{n} \mathcal{P}_{s}^{\eta_{s}}\left(y_{1}\right) \mathcal{P}_{p}^{\beta-\eta_{p}}\left(y_{1}\right) d y_{1} \\
& \quad+\frac{\mathcal{P}_{p}^{\beta-\eta_{p}}\left(z_{1}\right)}{\sigma^{\zeta} \Gamma(\zeta)} \int_{0}^{\varsigma} \frac{\exp \left[\frac{\sigma-1}{\sigma}\left(\varphi(\varsigma)-\varphi\left(y_{1}\right)\right)\right]}{\left(\varphi(\varsigma)-\varphi\left(y_{1}\right)\right)^{1-\zeta}} \varphi^{\prime}\left(y_{1}\right) \mathcal{R}\left(y_{1}\right) \prod_{s=1}^{n} \mathcal{P}_{s}^{\eta_{s}}\left(y_{1}\right) \mathcal{Q}^{\rho}\left(y_{1}\right) d y_{1} \\
& \geq \frac{\mathcal{Q}^{\rho}\left(z_{1}\right) \mathcal{P}_{p}^{\beta-\eta_{p}}\left(z_{1}\right)}{\sigma^{\zeta} \Gamma(\zeta)} \int_{0}^{\varsigma} \frac{\exp \left[\frac{\sigma-1}{\sigma}\left(\varphi(\varsigma)-\varphi\left(y_{1}\right)\right)\right]}{\left(\varphi(\varsigma)-\varphi\left(y_{1}\right)\right)^{1-\zeta}} \varphi^{\prime}\left(y_{1}\right) \mathcal{R}\left(y_{1}\right) \prod_{s=1}^{n} \mathcal{P}_{s}^{\eta_{s}}\left(y_{1}\right) d y_{1} \\
& \quad+\frac{1}{\sigma^{\zeta} \Gamma(\zeta)} \int_{0}^{\varsigma} \frac{\exp \left[\frac{\sigma-1}{\sigma}\left(\varphi(\varsigma)-\varphi\left(y_{1}\right)\right)\right]}{\left(\varphi(\varsigma)-\varphi\left(y_{1}\right)\right)^{1-\zeta}} \varphi^{\prime}\left(y_{1}\right) \mathcal{R}\left(y_{1}\right) \\
& \quad \times \prod_{s=1}^{n} \mathcal{P}_{s}^{\eta_{s}}\left(y_{1}\right) \mathcal{Q}^{\rho}\left(y_{1}\right) \mathcal{P}_{p}^{\beta-\eta_{p}}\left(z_{1}\right) d y_{1} .
\end{aligned}
$$

Now, in view of Definition 2.3, we get

$$
\begin{aligned}
\mathcal{Q}^{\rho}\left(z_{1}\right)^{\varphi} \mathcal{K}_{0^{+}}^{\zeta, \sigma}\left[\mathcal{R}(\varsigma) \mathcal{P}_{p}^{\beta}(\varsigma) \prod_{s \neq p}^{n} \mathcal{P}_{s}^{\eta_{s}}(\varsigma)\right] \\
+\mathcal{P}_{p}^{\beta-\eta_{p}}\left(z_{1}\right)^{\varphi} \mathcal{K}_{0^{+}}^{\zeta, \sigma}\left[\mathcal{R}(\varsigma) \mathcal{Q}^{\rho}(\varsigma) \prod_{s=1}^{n} \mathcal{P}_{s}^{\eta_{s}}(\varsigma)\right] \\
\geq \mathcal{Q}^{\rho}\left(z_{1}\right) \mathcal{P}_{p}^{\beta-\eta_{p}}\left(z_{1}\right)^{\varphi} \mathcal{K}_{0^{+}}^{\zeta, \sigma}\left[\mathcal{R}(\varsigma) \prod_{s \neq p}^{n} \mathcal{P}_{s}^{\eta_{s}}(\varsigma)\right] \\
+{ }^{\varphi} \mathcal{K}_{0^{+}}^{\zeta, \sigma}\left[\mathcal{R}(\varsigma) \mathcal{Q}^{\rho}(\varsigma) \mathcal{U}_{p}^{\beta}(\varsigma) \prod_{s=1}^{n} \mathcal{P}_{s}^{\eta_{s}}(\varsigma)\right] .
\end{aligned}
$$

Again, multiplying both sides of (3.18) by $\frac{1}{\sigma \zeta \Gamma(\zeta)} \frac{\exp \left[\frac{\sigma-1}{\sigma}\left(\varphi(\varsigma)-\varphi\left(z_{1}\right)\right)\right]}{\left.\left(\varphi(\varsigma)-\varphi\left(z_{1}\right)\right)\right)^{1-\zeta}} \varphi^{\prime}\left(z_{1}\right) \mathcal{R}\left(y_{1}\right) \prod_{s=1}^{n} \mathcal{P}_{s}^{\eta_{s}}\left(z_{1}\right)$, integrating the obtained inequality with respect to $z_{1}$ over $(0, \varsigma)$, and then using $(2.3)$, we obtain

$$
\begin{aligned}
& { }^{\varphi} \mathcal{K}_{0^{+}}^{\zeta, \sigma}\left[\mathcal{R}(\varsigma) \mathcal{P}_{p}^{\beta}(\varsigma) \prod_{s \neq p}^{n} \mathcal{P}_{s}^{\eta_{s}}(\varsigma)\right]{ }^{\varphi} \mathcal{K}_{0^{+}}^{\zeta, \sigma}\left[\mathcal{R}(\varsigma) \mathcal{Q}^{\rho}(\varsigma) \prod_{s=1}^{n} \mathcal{P}^{\eta_{s}}(\varsigma)\right] \\
& \geq{ }^{\varphi} \mathcal{K}_{0^{+}}^{\zeta, \sigma}\left[\mathcal{R}(\varsigma) \mathcal{Q}^{\rho}(\varsigma) \mathcal{P}_{p}^{\beta}(\varsigma) \prod_{s \neq p}^{n} \mathcal{P}_{s}^{\eta_{s}}(\varsigma)\right]{ }^{\varphi} \mathcal{K}_{0^{+}}^{\zeta, \sigma}\left[\mathcal{R}(\varsigma) \prod_{s=1}^{n} \mathcal{P}^{\eta_{s}}(\varsigma)\right],
\end{aligned}
$$

which is the desired inequality (3.15).

Theorem 3.11 Assume that two functions $\mathcal{P}_{s}(s=1,2, \ldots, n)$ and $\mathcal{Q}$ defined on $[0, \infty)$ are positive and continuous with the assumption that the function $\mathcal{R}:[0, \infty) \rightarrow \mathbb{R}$ is positive and continuous, function $\mathcal{Q}$ is increasing and function $\mathcal{P}_{s}(s=1,2, \ldots, n)$ is decreasing on 
$[0, \infty)$. Then for all $\varsigma>0$, the following inequality holds:

$$
\begin{aligned}
{ }^{\varphi} \mathcal{K}_{0^{+}}^{\zeta, \sigma}\left[\mathcal{R}(\varsigma) \mathcal{P}_{p}^{\beta}(\varsigma) \prod_{s \neq p}^{n} \mathcal{P}_{s}^{\eta_{s}}(\varsigma)\right]{ }^{\varphi} \mathcal{K}_{0^{+}}^{\vartheta, \sigma}\left[\mathcal{R}(\varsigma) \mathcal{Q}^{\rho}(\varsigma) \prod_{s=1}^{n} \mathcal{P}_{s}^{\eta_{s}}(\varsigma)\right] \\
+{ }^{\varphi} \mathcal{K}_{0^{+}}^{\vartheta, \sigma}\left[\mathcal{R}(\varsigma) \mathcal{P}_{p}^{\beta}(\varsigma) \prod_{s \neq p}^{n} \mathcal{P}_{s}^{\eta_{s}}(\varsigma)\right] \varphi \mathcal{K}_{0^{+}}^{\zeta, \sigma}\left[\mathcal{R}(\varsigma) \mathcal{Q}^{\rho}(\varsigma) \prod_{s=1}^{n} \mathcal{P}^{\eta_{s}}(\varsigma)\right] \\
\geq{ }^{\varphi} \mathcal{K}_{0^{+}}^{\vartheta, \sigma}\left[\mathcal{R}(\varsigma) \mathcal{Q}^{\rho}(\varsigma) \mathcal{P}_{p}^{\beta}(\varsigma) \prod_{s \neq p}^{n} \mathcal{P}_{s}^{\eta_{s}}(\varsigma)\right]{ }^{\varphi} \mathcal{K}_{0^{+}}^{\zeta, \sigma}\left[\mathcal{R}(\varsigma) \prod_{s=1}^{n} \mathcal{P}^{\eta_{s}}(\varsigma)\right] \\
+{ }^{\varphi} \mathcal{K}_{0^{+}}^{\vartheta, \sigma}\left[\mathcal{R}(\varsigma) \mathcal{Q}^{\rho}(\varsigma) \mathcal{P}_{p}^{\beta}(\varsigma) \prod_{s \neq p}^{n} \mathcal{P}_{s}^{\eta_{s}}(\varsigma)\right]{ }^{\varphi} \mathcal{K}_{0^{+}}^{\zeta, \sigma}\left[\mathcal{R}(\varsigma) \prod_{s=1}^{n} \mathcal{P}^{\eta_{s}}(\varsigma)\right],
\end{aligned}
$$

where $\beta \geq \eta_{p}>0, \rho>0, p=1,2, \ldots, n$ and $\sigma \in(0,1], \zeta \in \mathcal{C}, \mathfrak{R}(\zeta)>0$.

Proof To obtain the desired assertion (3.19), we multiply (3.18) by $\frac{1}{\sigma^{\vartheta} \Gamma(\vartheta)} \frac{\exp \left[\frac{\sigma-1}{\sigma}\left(\varphi(\varsigma)-\varphi\left(z_{1}\right)\right)\right]}{\left(\varphi(\varsigma)-\varphi\left(z_{1}\right)\right)^{1-\vartheta}} \times$ $\varphi^{\prime}\left(z_{1}\right) \mathcal{R}\left(z_{1}\right) \prod_{s=1}^{n} \mathcal{P}_{s}^{\eta_{s}}\left(z_{1}\right)$, where $\vartheta, \eta_{s}>0(s=1,2, \ldots, n), z_{1} \in(0, \varsigma), \varsigma>0$, and then integrate the obtained inequality with respect to $z_{1}$ over $(0, \varsigma)$ to get

$$
\begin{aligned}
& \frac{{ }^{\varphi} \mathcal{K}_{0^{+}}^{\zeta, \sigma}\left[\mathcal{R}(\varsigma) \mathcal{P}_{p}^{\beta}(\varsigma) \prod_{s \neq p}^{n} \mathcal{P}_{s}^{\eta_{s}}(\varsigma)\right]}{\sigma^{\vartheta} \Gamma(\vartheta)} \\
& \times \int_{0}^{\varsigma} \frac{\exp \left[\frac{\sigma-1}{\sigma}\left(\varphi(\varsigma)-\varphi\left(z_{1}\right)\right)\right]}{\left(\varphi(\varsigma)-\varphi\left(z_{1}\right)\right)^{1-\vartheta}} \varphi^{\prime}\left(z_{1}\right) \mathcal{R}\left(z_{1}\right) \prod_{s=1}^{n} \mathcal{P}_{s}^{\eta}\left(z_{1}\right) \mathcal{Q}^{\rho}\left(z_{1}\right) d z_{1} \\
& +\frac{{ }^{\varphi} \mathcal{K}_{0^{+}}^{\zeta, \sigma}\left[\mathcal{R}(\varsigma) \mathcal{Q}^{\rho}(\varsigma) \prod_{s=1}^{n} \mathcal{P}_{s}^{\eta_{s}}(\varsigma)\right]}{\sigma^{\vartheta} \Gamma(\vartheta)} \\
& \times \int_{0}^{\varsigma} \frac{\exp \left[\frac{\sigma-1}{\sigma}\left(\varphi(\varsigma)-\varphi\left(z_{1}\right)\right)\right]}{\left(\varphi(\varsigma)-\varphi\left(z_{1}\right)\right)^{1-\vartheta}} \varphi^{\prime}\left(z_{1}\right) \mathcal{R}\left(z_{1}\right) \prod_{s=1}^{n} \mathcal{P}_{s}^{\eta}\left(z_{1}\right) \mathcal{P}_{p}^{\beta-\eta_{p}}\left(z_{1}\right) d z_{1} \\
& \geq \frac{{ }^{\varphi} \mathcal{K}_{0^{+}}^{\zeta, \sigma}\left[\mathcal{R}(\varsigma) \prod_{s=1}^{n} \mathcal{P}_{s}^{\eta_{s}}(\varsigma)\right]}{\sigma^{\vartheta} \Gamma(\vartheta)} \\
& \times \int_{0}^{\varsigma} \frac{\exp \left[\frac{\sigma-1}{\sigma}\left(\varphi(\varsigma)-\varphi\left(z_{1}\right)\right)\right]}{\left(\varphi(\varsigma)-\varphi\left(z_{1}\right)\right)^{1-\vartheta}} \varphi^{\prime}\left(z_{1}\right) \mathcal{R}\left(z_{1}\right) \prod_{s=1}^{n} \mathcal{P}_{s}^{\eta}\left(z_{1}\right) \mathcal{Q}^{\rho}\left(z_{1}\right) \mathcal{P}_{p}^{\beta-\eta_{p}}\left(z_{1}\right) d z_{1} \\
& +\frac{{ }^{\varphi} \mathcal{K}_{0^{+}}^{\zeta, \sigma}\left[\mathcal{R}(\varsigma) \mathcal{Q}^{\rho}(\varsigma) \mathcal{P}^{\beta}(\varsigma) \prod_{s \neq p}^{n} \mathcal{P}_{s}^{\eta_{s}}(\varsigma)\right]}{\sigma^{\vartheta} \Gamma(\vartheta)} \\
& \times \int_{0}^{\varsigma} \frac{\exp \left[\frac{\sigma-1}{\sigma}\left(\varphi(\varsigma)-\varphi\left(z_{1}\right)\right)\right]}{\left(\varphi(\varsigma)-\varphi\left(z_{1}\right)\right)^{1-\vartheta}} \varphi^{\prime}\left(z_{1}\right) \mathcal{R}\left(z_{1}\right) \prod_{s=1}^{n} \mathcal{P}_{s}^{\eta}\left(z_{1}\right) d z_{1}
\end{aligned}
$$

It follows from Definition 2.3 that

$$
\begin{aligned}
{ }^{\varphi} \mathcal{K}_{0^{+}}^{\zeta, \sigma} & {\left[\mathcal{R}(\varsigma) \mathcal{P}_{p}^{\beta}(\varsigma) \prod_{s \neq p}^{n} \mathcal{P}_{s}^{\eta_{s}}(\varsigma)\right]{ }^{\varphi} \mathcal{K}_{0^{+}}^{\vartheta, \sigma}\left[\mathcal{R}(\varsigma) \mathcal{Q}^{\rho}(\varsigma) \prod_{s=1}^{n} \mathcal{P}^{\eta_{s}}(\varsigma)\right] } \\
& +{ }^{\varphi} \mathcal{K}_{0^{+}}^{\vartheta, \sigma}\left[\mathcal{R}(\varsigma) \mathcal{P}_{p}^{\beta}(\varsigma) \prod_{s \neq p}^{n} \mathcal{P}_{s}^{\eta_{s}}(\varsigma)\right]{ }^{\varphi} \mathcal{K}_{0^{+}}^{\zeta, \sigma}\left[\mathcal{R}(\varsigma) \mathcal{Q}^{\rho}(\varsigma) \prod_{s=1}^{n} \mathcal{P}^{\eta_{s}}(\varsigma)\right]
\end{aligned}
$$




$$
\begin{aligned}
\geq & { }^{\varphi} \mathcal{K}_{0^{+}}^{\zeta, \sigma}\left[\mathcal{R}(\varsigma) \mathcal{Q}^{\rho}(\varsigma) \mathcal{P}_{p}^{\beta}(\varsigma) \prod_{s \neq p}^{n} \mathcal{P}_{s}^{\eta_{s}}(\varsigma)\right]{ }^{\varphi} \mathcal{K}_{0^{+}}^{\vartheta, \sigma}\left[\mathcal{R}(\varsigma) \prod_{s=1}^{n} \mathcal{P}_{s}^{\eta_{s}}(\varsigma)\right] \\
& +{ }^{\varphi} \mathcal{K}_{0^{+}}^{\zeta, \sigma}\left[\mathcal{R}(\varsigma) \mathcal{Q}^{\rho}(\varsigma) \mathcal{P}_{p}^{\beta}(\varsigma) \prod_{s \neq p}^{n} \mathcal{P}_{s}^{\eta_{s}}(\varsigma)\right]{ }^{\varphi} \mathcal{K}_{0^{+}}^{\vartheta, \sigma}\left[\mathcal{R}(\varsigma) \prod_{s=1}^{n} \mathcal{P}_{s}^{\eta_{s}}(\varsigma)\right],
\end{aligned}
$$

which completes the proof of Theorem 3.11 .

Remark 3.12 Applying Theorem 3.11 for $\zeta=\vartheta$, we get Theorem 3.10.

\section{Conclusions}

In the article, we have derived certain variants by the use of the newly defined $\mathcal{G P \mathcal { F }}$ with respect to another function $\varphi$ related to a class of $n$ positive continuous and decreasing functions defined on $\left[\mu_{1}, \mu_{2}\right]$. In [76], Liu et al. investigated thought-provoking variants for continuous functions. Recently, Dahmani [77] has presented more generalizations of the work in [76] by utilizing the $\mathcal{R} \mathcal{L}$-fractional integral operators. Therefore our findings in the present article are generalizations of integral inequalities involving the $\mathcal{R} \mathcal{L}$-fractional integral operators. If we take into account $\mathcal{R}(\varsigma)=\mu=1$ and $\varphi(x)=x$, then our findings derived in the present paper will become variants associated with the $\mathcal{R} \mathcal{L}$-fractional integral operators introduced by Dahmani [77]. Particular cases of our consequences could be observed in [76]. The consequences acquired in this paper deliver some contributions to the direction of the idea of integral inequalities, fractional calculus, and anticipated results in some applications for establishing the uniqueness of solutions of integrodifferential equations and for finding the analytical solutions of some space-time fractional differential equations.

\section{Acknowledgements}

The authors would like to express their sincere thanks to the support of National Natural Science Foundation of China.

Funding

This work was supported by the National Natural Science Foundation of China (Grant No. 61673169).

Availability of data and materials

Not applicable.

Competing interests

The authors declare that they have no competing interests.

Authors' contributions

All authors contributed equally to the writing of this paper. All authors read and approved the final manuscript.

\section{Author details}

'Department of Mathematics and General Sciences, Prince Sultan University, Riyadh, Saudi Arabia. ${ }^{2}$ Department of Medical Research, China Medical University Taiwan, Taichung, Taiwan. ${ }^{3}$ Department of Computer Science and Information Engineering, Asia University, Taichung, Taiwan. ${ }^{4}$ Department of Mathematics, Government College University, Faisalabad, Pakistan. ${ }^{5}$ Department of Mathematics, Faculty of Science, Al-Azhar University, Cairo, Egypt. ${ }^{6}$ Département de Mathématiques, Faculté des Sciences et Techniques, Université Moulay Ismail, Errachidia, Morocco. ${ }^{7}$ Department of Mathematics, Huzhou University, Huzhou, China. ${ }^{8}$ Hunan Provincial Key Laboratory of Mathematical Modeling and Analysis in Engineering, Changsha University of Science \& Technology, Changsha, China.

\section{Publisher's Note}

Springer Nature remains neutral with regard to jurisdictional claims in published maps and institutional affiliations. 


\section{References}

1. Cattani, C.: Haar wavelet-based technique for sharp jumps classification. Math. Comput. Model. 39(2-3), 255-278 (2004)

2. Cattani, C.: A review on harmonic wavelets and their fractional extension. J. Adv. Eng. Comput. 2(4), 224-238 (2018)

3. Valliammal, N., Ravichandran, C., Hammouch, Z., Baskonus, H.M.: A new investigation on fractional-order neutra differential systems with state-dependent delay. Int. J. Nonlinear Sci. Numer. Simul. 20(7-8), 803-809 (2019)

4. Shen, J.-M., Rashid, S., Noor, M.A., Ashraf, R., Chu, Y.-M.: Certain novel estimates within fractional calculus theory on time scales. AIMS Math. 5(6), 6073-6086 (2020)

5. Khurshid, Y., Adil Khan, M., Chu, Y.-M.: Conformable fractional integral inequalities for GG- and GA-convex functions. AIMS Math. 5(5), 5012-5030 (2020)

6. Chu, Y.-M., Awan, M.U., Javad, M.Z., Khan, A.W.: Bounds for the remainder in Simpson's inequality via $n$-polynomial convex functions of higher order using Katugampola fractional integrals. J. Math. 2020, Article ID 4189036 (2020)

7. Yan, P.-Y., Li, Q., Chu, Y.-M., Mukhtar, S., Waheed, S.: On some fractional integral inequalities for generalized strongly modified $h$-convex function. AIMS Math. 5(6), 6620-6638 (2020)

8. Rashid, S., Jarad, F., Chu, Y.-M.: A note on reverse Minkowski inequality via generalized proportional fractional integral operator with respect to another function. Math. Probl. Eng. 2020, Article ID 7630260 (2020)

9. Pratap, A., Raja, R., Alzabut, J., Cao, J.-D., Rajchakit, G., Huang, C.-X.: Mittag-Leffler stability and adaptive impulsive synchronization of fractional order neural networks in quaternion field. Math. Methods Appl. Sci. 43(10), 6223-6253 (2020)

10. Zhou, S.-S., Rashid, S., Jarad, F., Kalsoom, H., Chu, Y.-M.: New estimates considering the generalized proportional Hadamard fractional integral operators. Adv. Differ. Equ. 2020, Article ID 275 (2020)

11. Xu, L., Chu, Y.-M., Rashid, S., El-Deeb, A.A., Nisar, K.S.: On new unified bounds for a family of functions with fractional q-calculus theory. J. Funct. Spaces 2020, Article ID 4984612 (2020)

12. Huang, C.-X., Liu, L.-Z.: Sharp function inequalities and boundedness for Toeplitz type operator related to general fractional singular integral operator. Publ. Inst. Math. 92(106), 165-176 (2012)

13. Awan, M.U., Talib, S., Chu, Y.-M., Noor, M.A., Noor, K.I.: Some new refinements of Hermite-Hadamard-type inequalities involving $\Psi_{k}$-Riemann-Liouville fractional integrals and applications. Math. Probl. Eng. 2020, Article ID 3051920 (2020)

14. Yang, X.-Z., Farid, G., Nazeer, W., Chu, Y.-M., Dong, C.-F.: Fractional generalized Hadamard and Fejér-Hadamard inequalities for $m$-convex function. AIMS Math. 5(6), 6325-6340 (2020)

15. Guo, S.-Y., Chu, Y.-M., Farid, G., Mehmood, S., Nazeer, W.: Fractional Hadamard and Fejér-Hadamard inequalities associated with exponentially $(s, m)$-convex functions. J. Funct. Spaces 2020, Article ID 2410385 (2020)

16. Rashid, S., Khalid, A., Rahman, S., Nisar, K.S., Chu, Y.-M.: On new modifications governed by quantum Hahn's integral operator pertaining to fractional calculus. J. Funct. Spaces 2020, Article ID 8262860 (2020)

17. Huang, C.-X., Zhang, H., Huang, L.-H.: Almost periodicity analysis for a delayed Nicholson's blowflies model with nonlinear density-dependent mortality term. Commun. Pure Appl. Anal. 18(6), 3337-3349 (2019)

18. Iqbal, A., Adil Khan, M., Ullah, S., Chu, Y.-M.: Some new Hermite-Hadamard-type inequalities associated with conformable fractional integrals and their applications. J. Funct. Spaces 2020, Article ID 9845407 (2020)

19. Khurshid, Y., Adil Khan, M., Chu, Y.-M.: Conformable integral version of Hermite-Hadamard-Fejér inequalities via $\eta$-convex functions. AIMS Math. 5(5), 5106-5120 (2020)

20. Qi, H.-X., Yussouf, M., Mehmood, S., Chu, Y.-M., Farid, G.: Fractional integral versions of Hermite-Hadamard type inequality for generalized exponentially convexity. AIMS Math. 5(6), 6030-6042 (2020)

21. Zhang, Y., Cattani, C., Yang, X.-J.: Local fractional homotopy perturbation method for solving non-homogeneous heat conduction equations in fractal domains. Entropy 17(10), 6753-6764 (2015)

22. Kumar, D., Singh, J., Baleanu, D., Rathore, S.: Analysis of regularized long-wave equation associated with a new fractional operator with Mittag-Leffer type kernel. Physica A 492, 155-167 (2018)

23. Singh, J., Kumar, D., Hammouch, Z., Atangana, A.: A fractional epidemiological model for computer viruses pertaining to a new fractional derivative. Appl. Math. Comput. 316, 504-515 (2018)

24. Owolabi, K.M., Hammouch, Z.: Spatiotemporal patterns in the Belousov-Zhabotinskii reaction systems with Atangana-Baleanu fractional order derivative. Physica A 523, 1072-1090 (2019)

25. Adil Khan, M., Hanif, M., Khan, Z.A., Ahmad, K., Chu, Y.-M.: Association of Jensen's inequality for s-convex function with Csiszár divergence. J. Inequal. Appl. 2019, Article ID 162 (2019)

26. Jothimani, K., Kaliraj, K., Hammouch, Z., Ravichandran, C.: New results on controllability in the framework of fractional integrodifferential equations with nondense domain. Eur. Phys. J. Plus 134, Article ID 441 (2019)

27. Rashid, S., Ashraf, R., Noor, M.A., Noor, K.I., Chu, Y.-M.: New weighted generalizations for differentiable exponentially convex mapping with application. AIMS Math. 5(4), 3525-3546 (2020)

28. Ge-JiLe, H., Rashid, S., Noor, M.A., Suhail, A., Chu, Y.-M.: Some unified bounds for exponentially tgs-convex functions governed by conformable fractional operators. AIMS Math. 5(6), 6108-6123 (2020)

29. Atangana, A.: Modelling the spread of COVID-19 with new fractal-fractional operators: can the lockdown save mankind before vaccination? Chaos Solitons Fractals 136, Article ID 109860 (2020)

30. Doungmo, G., Emile, F., Khan, Y., Chaudhry, Q.A.: HIV and shifting epicenters for COVID-19, an alert for some countries. Chaos Solitons Fractals 139, Article ID 110030 (2020)

31. Wang, J.-F., Huang, C.-X., Huang, L.-H.: Discontinuity-induced limit cycles in a general planar piecewise linear system of saddle-focus type. Nonlinear Anal. Hybrid Syst. 33, 162-178 (2019)

32. Khan, S., Adil Khan, M., Chu, Y.-M.: Converses of the Jensen inequality derived from the Green functions with applications in information theory. Math. Methods Appl. Sci. 43(5), 2577-2587 (2020)

33. Khan, M.A., Atangana, A.: Modeling the dynamics of novel coronavirus (2019-nCov) with fractional derivative. Alex. Eng. J. (2020). https://doi.org/10.1016/j.aej.2020.02.033

34. Danane, J., Allali, K., Hammouch, Z:: Mathematical analysis of a fractional differential model of HBV infection with antibody immune response. Chaos Solitons Fractals 136, Article ID 109787 (2020)

35. Gao, W., Veeresha, P., Baskonus, H.M., Prakasha, D.G., Kumar, P.: A new study of unreported cases of 2019-nCOV epidemic outbreaks. Chaos Solitons Fractals 138, Article ID 109929 (2020) 
36. Losada, J., Nieto, J.J.: Properties of a new fractional derivative without singular kernel. Prog. Fract. Differ. Appl. 1(2), 87-92 (2015)

37. O'Regan, D., Samet, B.: Lyapunov-type inequalities for a class of fractional differential equations. J. Inequal. Appl. 2015, Article ID 247 (2015)

38. Owolabi, K.M.: Numerical approach to fractional blow-up equations with Atangana-Baleanu derivative in Riemann-Liouville sense. Math. Model. Nat. Phenom. 13(1), Article ID 7 (2018)

39. Mohammad, M., Trounev, A., Cattani, C.: The dynamics of COVID-19 in the UAE based on fractional derivative modeling using Riesz wavelets simulation. https://assets.researchsquare.com/files/rs-33366/v1_stamped.pdf

40. IIhan, E., Kıymaz, I.O.: A generalization of truncated M-fractional derivative and applications to fractional differential equations. Appl. Math. Nonlinear Sci. 5(1), 171-188 (2020)

41. Gao, W., Veeresha, P., Prakasha, D.G., Baskonus, H.M.: Novel dynamic structures of 2019-nCoV with nonlocal operator via powerful computational technique. Biology 9, Article ID 107 (2020)

42. Zhao, T.-H., He, Z.-Y., Chu, Y.-M.: On some refinements for inequalities involving zero-balanced hypergeometric function. AIMS Math. 5(6), 6479-6495 (2020)

43. Atangana, A., Iğret, S.: Mathematical model of COVID-19 spread in Turkey and South Africa: theory, methods and applications. https://doi.org/10.1101/2020.05.08.20095588

44. Abdeljawad, T., Baleanu, D.: On fractional derivatives with exponential kernel and their discrete versions. Rep. Math. Phys. 80(1), 11-27 (2017)

45. Huang, C.-X., Liu, L.-Z.: Boundedness of multilinear singular integral operator with a non-smooth kernel and mean oscillation. Quaest. Math. 40(3), 295-312 (2017)

46. Almeida, R.: A Caputo fractional derivative of a function with respect to another function. Commun. Nonlinear Sci. Numer. Simul. 44, 460-481 (2017)

47. Baleanu, D., Diethelm, K., Scalas, E., Trujillo, J.J.: Fractional Calculus. World Scientific, Hackensack (2012)

48. Jarad, F., Uğurlu, E., Abdeljawad, T., Baleanu, D.: On a new class of fractional operators. Adv. Differ. Equ. 2017, Article ID $247(2017)$

49. Miller, K.S., Ross, B.: An Introduction to the Fractional Calculus and Fractional Differential Equations. Wiley, New York (1993)

50. Podlubny, I.: Fractional Differential Equations. Academic Press, San Diego (1999)

51. Abdeljawad, T.: On conformable fractional calculus. J. Comput. Appl. Math. 279, 57-66 (2015)

52. Alzabut, J., Abdeljawad, T., Jarad, F., Sudsutad, W.: A Gronwall inequality via the generalized proportional fractional derivative with applications. J. Inequal. Appl. 2019, Article ID 101 (2019)

53. Jarad, F., Abdeljawad, T., Alzabut, J.: Generalized fractional derivatives generated by a class of local proportional derivatives. Eur. Phys. J. Spec. Top. 226, 3457-3471 (2017)

54. Jarad, F., Alqudah, M.A., Abdeljawad, T.: On more general forms of proportional fractional operators. Open Math. 18(1), 167-176 (2020)

55. Rashid, S., Jarad, F., Noor, M.A., Kalsoom, H., Chu, Y.-M.: Inequalities by means of generalized proportional fractional integral operators with respect to another function. Mathematics 7(12), Article ID 1225 (2019)

56. Agarwal, P., Kadakal, M., Işcan, I., Chu, Y.-M.: Better approaches for $n$-times differentiable convex functions. Mathematics 8, Article ID 950 (2020)

57. Wang, M.-K., Chu, H.-H., Li, Y.-M., Chu, Y.-M.: Answers to three conjectures on convexity of three functions involving complete elliptic integrals of the first kind. Appl. Anal. Discrete Math. 14(1), 255-271 (2020)

58. Shen, J.-M., Yang, Z.-H., Qian, W.-M., Zhang, W., Chu, Y.-M.: Sharp rational bounds for the gamma function. Math. Inequal. Appl. 23(3), 843-853 (2020)

59. Wang, M.-K., Chu, Y.-M., Li, Y.-M., Zhang, W.: Asymptotic expansion and bounds for complete elliptic integrals. Math. Inequal. Appl. 23(3), 821-841 (2020)

60. Hai, G.-J., Zhao, T.-H.: Monotonicity properties and bounds involving the two-parameter generalized Grötzsch ring function. J. Inequal. Appl. 2020, Article ID 66 (2020)

61. Awan, M.U., Akhtar, N., Kashuri, A., Noor, M.A., Chu, Y.-M.: 2D approximately reciprocal $\rho$-convex functions and associated integral inequalities. AIMS Math. 5(5), 4662-4680 (2020)

62. Sun, M.-B., Chu, Y.-M.: Inequalities for the generalized weighted mean values of $g$-convex functions with applications. Rev. R. Acad. Cienc. Exactas Fís. Nat., Ser. A Mat. 114(4), Article ID 172 (2020)

63. Abbas Baloch, I., Mughal, A.A., Chu, Y.-M., Haq, A.U., De La Sen, M.: A variant of Jensen-type inequality and related results for harmonic convex functions. AIMS Math. 5(6), 6404-6418 (2020)

64. Adil Khan, M., Pečarić, J., Chu, Y.-M.: Refinements of Jensen's and McShane's inequalities with applications. AIMS Math. 5(5), 4931-4945 (2020)

65. Zhao, T.-H., Wang, M.-K., Chu, Y.-M.: A sharp double inequality involving generalized complete elliptic integral of the first kind. AIMS Math. 5(5), 4512-4528 (2020)

66. Kalsoom, H., Idrees, M., Baleanu, D., Chu, Y.-M.: New estimates of $q_{1} q_{2}$-Ostrowski-type inequalities within a class of n-polynomial prevexity of function. J. Funct. Spaces 2020, Article ID 3720798 (2020)

67. Iqbal, A., Adil Khan, M., Mohammad, N., Nwaeze, E.R., Chu, Y.-M.: Revisiting the Hermite-Hadamard integral inequality via a Green function. AIMS Math. 5(6), 6087-6107 (2020)

68. Awan, M.U., Akhtar, N., Iftikhar, S., Noor, M.A., Chu, Y.-M.: New Hermite-Hadamard type inequalities for n-polynomial harmonically convex functions. J. Inequal. Appl. 2020, Article ID 125 (2020)

69. Awan, M.U., Talib, S., Noor, M.A., Chu, Y.-M., Noor, K.I.: Some trapezium-like inequalities involving functions having strongly n-polynomial preinvexity property of higher order. J. Funct. Spaces 2020. Article ID 9154139 (2020)

70. Khalil, R., Al Horani, M., Yousef, A., Sababheh, M.: A new definition of fractional derivative. J. Comput. Appl. Math. 264, 65-70 (2014)

71. Rahman, G., Abdeljawad, T., Khan, A., Nisar, K.S.: Some fractional proportional integral inequalities. J. Inequal. Appl. 2019, Article ID 244 (2019)

72. Rashid, S., Safdar, F., Akdemir, A., Noor, M.A., Noor, K.I.: Some new fractional integral inequalities for exponentially m-convex functions via extended generalized Mittag-Leffler function. J. Inequal. Appl. 2019, Article ID 299 (2019)

73. Kilbas, A.A., Srivastava, H.M., Trujillo, J.J.: Theory and Applications of Fractional Differential Equations. Elsevier, Amsterdam (2006) 
74. Rahman, G., Abdeljawad, T., Jarad, F., Khan, A., Nisar, K.S.: Certain inequalities via generalized proportional Hadamard fractional integral operators. Adv. Differ. Equ. 2019, Article ID 454 (2019)

75. Samko, S.G., Kilbas, A.A., Marichev, O.I.: Fractional Integrals and Derivatives. Gordon \& Breach, Yverdon (1993)

76. Liu, W.-J., Ngô, Q.-A., Huy, V.N.: Several interesting integral inequalities. J. Math. Inequal. 3(2), 201-212 (2009)

77. Dahmani, Z: New classes of integral inequalities of fractional order. Matematiche 69(1), 237-247 (2014)

Submit your manuscript to a SpringerOpen ${ }^{\odot}$ journal and benefit from:

- Convenient online submission

Rigorous peer review

Open access: articles freely available online

- High visibility within the field

- Retaining the copyright to your article

Submit your next manuscript at $\gg$ springeropen.com 